\title{
Sedimentuntersuchungen an unterpleistozänen Schmelzwasserablagerungen und Periglazialschottern im Riß-Iller-Gebiet, deutsches Alpenvorland
}

\author{
Andreas Gerth \& Raimo Becker-HaumanN ${ }^{*}$
}

Keywords: Lower Pleistocene, Paleogeography, Stratigraphy, Glacifluvial gravel, Periglacial gravel, Petrographic analysis, Heavy mineral analyses, Germany, Alpine Foreland

Kurzfassung: Die unterpleistozänen Schmelzwassersedimente im Übergangsbereich von Rhein- und Illergletscher (Baden-Württemberg, Bayern) sowie die darunter anstehenden Sedimente periglazialer Schüttungen (Ältester Periglazialschotter) werden in dieser Arbeit beschrieben. Im Vordergrund steht die geröllpetrographische und schwermineralogische Untersuchung der Sedimente aus dem Biber-, Donau- und GünzGlazial. Die Ergebnisse bestätigen, dass die bereits von früheren Bearbeitern vorgenommene Trennung des Ältesten Periglazialschotters von den darüber lagernden Schmelzwassersedimenten aufgrund des Bestandes kristalliner Gerölle sowie auf Grund differierender Schwermineralvergesellschaftungen sicher möglich ist. Durch die hohe Beprobungsdichte ist innerhalb der glazifluviatilen Schotterniveaus eine kontinuierliche Änderung der petrographischen Zusammensetzung erkennbar. In der Geröllfraktion steigt zum Hangenden der Anteil kristalliner Gerölle und der so genannten Harten (Radiolarit, Quarzit), während sich die Karbonate verringern. Die Daten weisen darauf hin, dass es sich hierbei um eine Veränderung des Geröllspektrums und nicht um einen Verwitterungseffekt handelt. Bei den Schwermineralen sinken die Gehalte an instabilen Mineralen (Granat, Hornblende) ab, hingegen steigt der Gehalt an Staurolith zu den jüngeren Ablagerungen stark an. Die qualitativen Ergebnisse werden in den Kontext der rekonstruierten Paläo-Talfüllungen gestellt und die flussgeschichtliche Entwicklung des Riß-Iller-Gebietes nachgezeichnet.

[Sedimentological research on Lower Pleistocene meltwater deposits and periglacial sediments of the Rhine-Iller area; German Alpine Foreland]

\begin{abstract}
The Lower Pleistocene meltwater deposits at the contact of the Rhine- and Illerglacier (BadenWürttemberg, Bavaria) and the periglacial sediments occurring below them are described. The research is focused on the analysis of the petrography of the pebble-size fraction and of the sand-size heavy mineral assemblages of the Biber-, Donau- and Günz-age deposits. The results confirm that the periglacial sediments can be distinguished clearly from the glaciofluvial material by arguments of the petrography. However, a systematic change of the lithology also within the glaciofluvial material can be inferred from the high number of the investigated samples. Within the gravel fraction the amount of crystalline rocks, radiolarite and hornstone increases towards the younger accumulation units, whereas the content of calcareous components decreases. Concerning the heavy minerals the amount of instable minerals as Garnet and Hornblende decreases, while the portion of Staurolite increases remarkably towards the younger deposits. In the paper at hand these results are interpreted with respect to the palaeogeography, in order to figure out the river development of the Riß-Iller-tract.
\end{abstract}

* Anschriften der Verfasser: Dipl.-Geol. A. Gerth, PD Dr. R. Becker-Haumann, Geographisches Institut der Universität zu Köln, Albertus-Magnus-Platz, 50923 Köln, r.behaumann@uni-koeln.de 


\section{Einführung}

Die Riß-Iller-Platte ist ein klassisches Untersuchungsgebiet für glaziale und glazifluviatile Ablagerungen im Übergangsbereich des östlichen Rhein- sowie des westlichen Illergletschers. Insbesondere die haslachbis würmzeitlichen Schotter wurden in der bisherigen Literatur eingehenden sedimentologisch-petrographischen Untersuchungen unterzogen, um eine zeitliche und flussgeschichtliche Gliederung aufzustellen. Aufgrund schlechter Erhaltungsbedingungen sind die älteren Schottervorkommen bislang in nur wenigen Beiträgen - so grundlegend von SINN (1972) und LöSCHER (1976) sowie zuletzt von Doppler (2003) und Villinger (2003) - beschrieben worden. Es bot sich daher an, im Iller-Riß-Gebiet diese Ablagerungen eingehend $\mathrm{zu}$ untersuchen und sie mit den Sedimenten der Nachbargebiete zu vergleichen. Dazu war es hilfreich, dass in BECKER-HAumann (2005) für das Illergletscher-Vorland ein stratigraphisches und paläogeographisches Konzept dargestellt wird, mit dem sich die neuen Befunde abgleichen ließen.

Um eine Konnektion beider Gebiete zu ermöglichen, ist die Riß-Iller-Platte im Rahmen einer separaten Kampagne geologisch kartiert, die Ablagerungen beprobt und der Datensatz GIS-gestützt verwaltet worden. Zudem ist die stratigraphische Gliederung und die flussgeschichtliche Einstufung der Schmelzwassersediment-Abfolgen überprüft worden. Die Ergebnisse des Projekts ergänzen die Arbeit von Doppler (2003), wobei insbesondere die GIS-gestützte 3D-Modellierung neue Ergebnisse für die flussgeschichtliche Interpretation der Ablagerungen erbracht hat. Die ehemaligen Sedimentfüllungen der Schmelzwassertäler wurden über ihre Oberund Basisflächen definiert und unter Berücksichtigung hydraulischer Randbedingungen sowie der Talgeometrie dreidimensional modelliert. Die methodischen Grundlagen sind ausführlich in BECKER-HAumANn (2007), die Resultate im Bezug auf das Riß-Iller-Gebiet in Becker-Haumann \& Gerth (im Druck) erläutert. Auf die Modellierungsergebnisse wird hier nur im Hinblick auf die flussgeschichtli- che Entwicklung Bezug genommen (Kap. 4). In vorliegender Arbeit werden die neu gewonnenen petrographischen und sedimentologischen Daten für die biber- bis günzzeitlichen Schmelzwassersedimente vorgestellt. Das Untersuchungsgebiet liegt zwischen den Städten Ulm im Norden und Memmingen im Süden (Abb. 1). Im Nordwesten wird es vom Tal der Donau, im Westen und Osten vom Tal der Roth begrenzt. Im Süden schließen sich die Anhöhen mehrerer Endmoränenzüge an. Die untersuchten Schottervorkommen weisen insgesamt eine Fläche von rund $112 \mathrm{~km}^{2}$ auf.

Für das Riß-Iller-Gebiet existieren zahlreiche stratigraphische Gliederungen und Alterszuordnungen der pleistozänen Ablagerungen (Tab. 1), aus denen sich eine Terrassenstratigraphie der Region ergibt (Tab. 2). So werden die Schottervorkommen des Erlenmooser und Erolzheimer Riedels, des Heggbacher und des Holzstöcke-Feldes (Abb. 1) von Penck \& BRÜCKNer (1901-1909) als Ältere Deckenschotter in das Günzglazial gestellt. Weidenbach $(1937,1940)$ und Schädel (1952) unterstützen diese Zuordnung des Erlenmooser und Erolzheimer Riedels. Die Hochflächen des Holzstöcke- und Heggbacher Feldes werden von den letztgenannten Bearbeitern in das Mindelglazial gestellt. Der Erlenmooser und Erolzheimer Schotterriedel werden seit GRAUL (1962, 1968) als donauzeitlich eingeordnet. Das von ihm als donauzeitlich ausgewiesene Heggbacher Feld wird von SCHÄDEL \& WERNER (1963) als günzzeitlich angesehen. Das Holzstöcke-Feld wird von GraUL (1962) dem Günz zugeordnet. Diese stratigraphische Zuordnung wird in der Literatur bis heute eingehalten, ebenso wie die Stellung des Kellmünzer Feldes in das Donauglazial (Löscher 1976) und die der Walpertshofener Schotteranhöhe in das Günz (HAAG 1982). Die Schotterkörper von Illerrieden und Dorndorf werden von SCHÄDEL (1952) in das Mindel I und Günz II gestellt. GraUL (1962) sieht den Illerriedener Schotterkörper als Donau I-zeitlich, die südliche Dorndorfer Schotterfläche jedoch als Donau II-zeitlich an. Dessen nördlicher Bereich wird von ihm dem Günz I zugeordnet. Beide Vorkommen werden von SCHÄDEL \& WERnER (1963) hingegen zum Donau II gerechnet. 


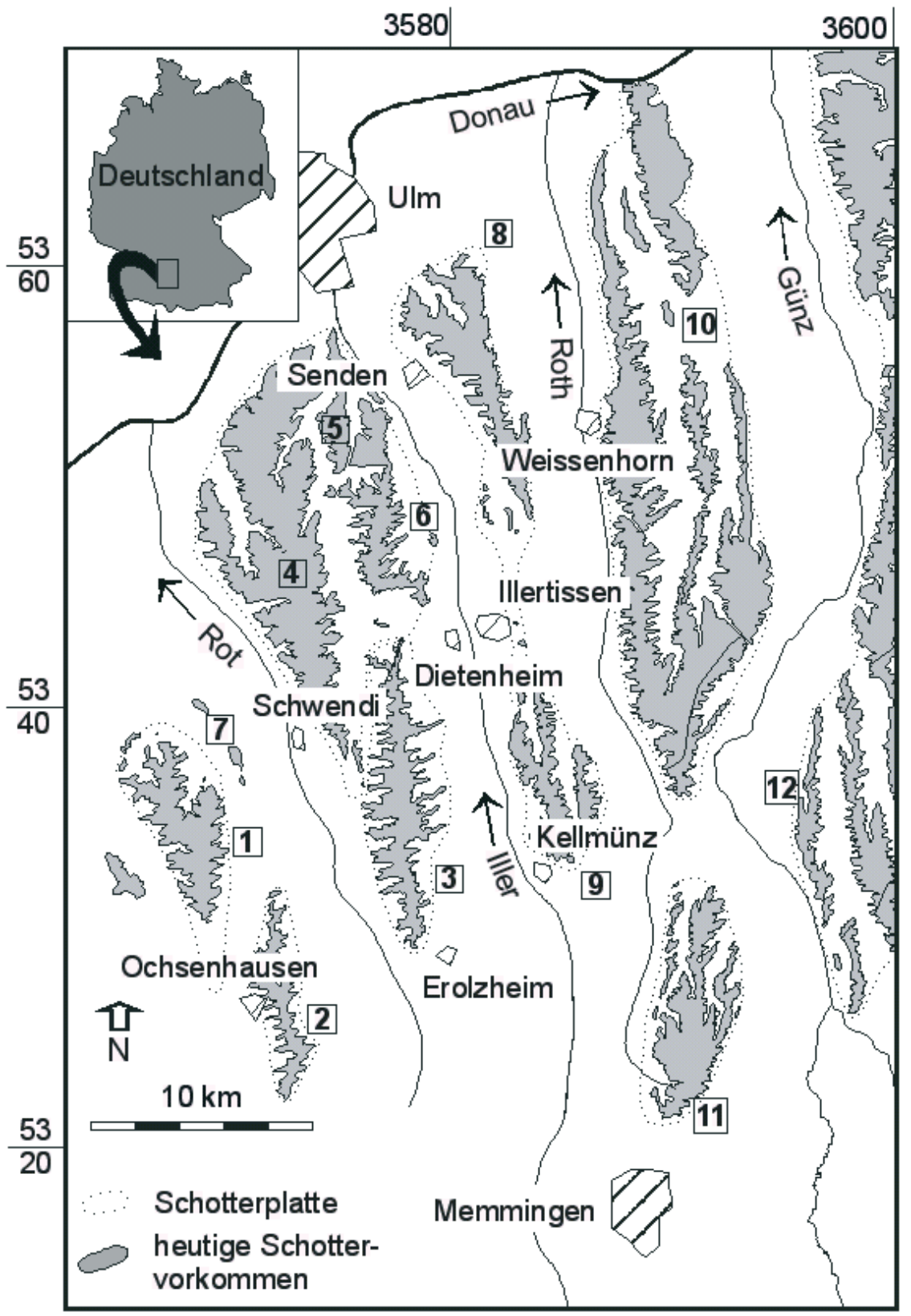

Abb. 1: Lage des Arbeitsgebietes mit den untersuchten Schottervorkommen 1 bis 6 sowie angrenzenden Schotterkörpern. Es bedeuten 1: Heggbacher Feld, 2: Erlenmooser Riedel, 3: Erolzheimer Riedel, 4: Holzstöcke-Feld, 5: Sendener Feld, 6: Kellmünzer Feld, 7: Weißenhorn-Kissendorfer Feld, 8: Eisenburger Feld, 9: Inneberg-Reisensburger Feld.

Fig. 1: General map showing the investigated gravel occurrences 1 to 6 and gravel areas in the neighbourhood. 
Tab. 1: Zusammenstellung der stratigraphischen Gliederungen für die untersuchten Schotterkörper aus wichtigen Bearbeitungen. Die Gebiete von Weißenhorn-Kissendorfer Feld (Nr. 7 in Abb. 1), Eisenburger Feld (Nr. 8) und Inneberg-Reisensburger Feld (Nr. 9) sind im Rahmen der vorliegenden Arbeit nicht neu aufgenommen worden. ÄDS: Älterer Deckenschotter; JDS - Jüngerer Deckenschotter; UZT, MZT, OZT - Untere, mittlere, obere Zwischenterrassenschotter; UDS: Untere Deckschotter, ÄP - Ältestpleistozän; PGS - Periglaziale Schotter.

Tab. 1: Summary of stratigraphies for the investigated gravel areas derived from important papers. Abbreviations see above.

\begin{tabular}{|c|c|c|c|c|c|c|c|c|}
\hline \multicolumn{9}{|c|}{ Schotterkörper (Nummerierung nach Abbildung 1) } \\
\hline Bearbeiter & 1 (Süd) & 1 (Nord) & 2 & 3 & 4 & 5 (Süd) & 5 (Nord) & 6 \\
\hline $\begin{array}{c}\text { PENCK \& } \\
\text { BRÜCKNER }\end{array}$ & ÄDS & - & ÄDS & ÄDS & ÄDS & - & - & - \\
\hline (1901-1909) & (Günz) & & (Günz) & (Günz) & (Günz) & & & \\
\hline WEIDENBACH & JDS & - & Günz & Günz & JDS & - & - & - \\
\hline$(1937 / 1940)$ & (Mindel) & & & & (Mindel) & & & \\
\hline $\begin{array}{l}\text { Übersichtskart. } \\
\text { SW- }\end{array}$ & Mindel & - & Günz & Günz & Mindel & Günz & Günz & Günz \\
\hline \multicolumn{9}{|l|}{ Deutschl. (1938) } \\
\hline SCHÄDEL (1953) & Mindel I & Mindel I & Günz I & Günz I & Mindel I & Günz II & Mindel I & - \\
\hline \multirow[t]{2}{*}{ GRAUL(1962) } & Donau II & Donau II & Donau I & Donau I & Günz I & Donau I & Donau II + & - \\
\hline & & & & & & & Günz I & \\
\hline $\begin{array}{l}\text { SCHÄDEL \& } \\
\text { WERNER }\end{array}$ & Günz & - & Donau I & Donau I & Günz & Donau II & Donau II & - \\
\hline \multicolumn{9}{|l|}{ (1963) } \\
\hline GRAUL (1968) & Donau II & - & Donau I & Donau I & Günz & Donau II & Donau II & - \\
\hline \multirow[t]{3}{*}{ LÖSCHER (1976) } & - & - & UDS & UDS & UZT & MZT & MZT/OZT & UDS \\
\hline & & & (Donau) & (Donau) & (Günz) & (Günz) & Günz & (Donau) \\
\hline & & & + PGS & + PGS & & & & + PGS \\
\hline \multirow[t]{2}{*}{ HAAG(1982) } & Günz & Günz & ÄP & ÄP & Günz & - & - & ÄP \\
\hline & & & (Donau) & (Donau) & & & & (Donau) \\
\hline Übersichtskarte & Günz & Günz & Donau & Donau & Günz & Günz & Günz & Donau \\
\hline \multicolumn{9}{|l|}{$\begin{array}{c}\text { Iller-Riß-Gebiet } \\
\text { (1988) }\end{array}$} \\
\hline $\begin{array}{c}\text { SCHREINER } \\
(1989 \mathrm{ff})\end{array}$ & Günz & Günz & Donau & Donau & Günz & Günz & Günz & Donau \\
\hline \multirow[t]{2}{*}{ DOPPLER (2003) } & Günz 2 & Günz 2 & Donau+Günz & Donau+Günz & Günz 2 & $\begin{array}{c}\text { Günz } \\
1+2 \\
\end{array}$ & Günz 1+2 & Donau+Günz \\
\hline & & & $+P G S$ & + PGS & & & & + PGS \\
\hline
\end{tabular}

LÖSCHER (1976) stellt das Holzstöcke-Feld, die Illerriedener und Dorndorfer Schotterkörper zu unterschiedlichen Niveaus von Zwischenterrassenschottern, die zum größten Teil dem Günz zugeordnet werden. Auf Grund des Fehlens von Dolomitgeröllen, die für glazifluviatile Ablagerungen des Nördlichen Alpenvorlands typisch sind, sollen sie durch periglazial-fluviatile Umlagerung entstanden sein. Die donauzeitlichen, kristallinarmen und teilweise dolomitführenden Schotter von Och- senhausen, Erolzheim und Kellmünz werden von LöSCHER (1976) als Hangendfazies mit glazifluviatiler Genese bezeichnet. Die ebenfalls von Löscher (1976) im Riß-Iller-Gebiet beschriebene kristallinreiche, dolomitfreie Liegendfazies - von Doppler (2003) als Älteste Periglazialschotter in Molassekristallinfazies bezeichnet - steht unter den als donauzeitlich eingestuften Sedimenten an. Sie ist nach SinN (1972) ein Aufarbeitungsprodukt aus der Adelegg, das von einer periglazialen 
Tab. 2: Terrassenstratigraphische Gliederungen des Iller-Riss-Gebietes nach den Vorstellungen von GraUL (1943, 1949, 1962), Penck \& BrüCKNer (1901-1909) und Jerz (1975).

Tab. 2: Stratigraphies of the terrace succession of the Iller-Riss-tract according to GRAUL $(1943,1949,1962)$, PENCK \& BRÜCKNER (1901-1909) and JeRZ (1975).

\begin{tabular}{|c|c|c|}
\hline System I & System II & Stratigraphie \\
\hline Niederterrassenschotter & \begin{tabular}{|} 
Niederterrassenschotter \\
Tieferer Niederterrassenschotter \\
Höherer Niederterrassenschotter
\end{tabular} & Würm \\
\hline $\begin{array}{r}\text { Hochterrassenschotter } \\
\text { Tieferes Niveau } \\
\text { Höheres Niveau }\end{array}$ & $\begin{array}{r}\text { Hochterrassenschotter } \\
\text { Tieferer Hochterrassenschotter } \\
\text { Höherer Hochterrassenschotter }\end{array}$ & Riss \\
\hline $\begin{array}{l}\text { Zwischenterrassenschotter } \\
\qquad \begin{array}{l}\text { Unterer Zwischenterrassenschotter } \\
\text { Mittlerer Zwischenterrassenschotter }\end{array}\end{array}$ & \begin{tabular}{|} 
Jüngerer Deckenschotter \\
Tieferer Jüngerer Deckenschotter \\
Höherer jüngerer Deckenschotter
\end{tabular} & $\begin{array}{l}\text { Mindel / Günz } \\
\text { Mindel / Haslach } \\
\text { Mindel } \\
\text { Haslach }\end{array}$ \\
\hline Oberer Zwischenterrassenschotter* & \multirow[t]{2}{*}{$\begin{array}{r}\text { Älterer Deckenschotter } \\
\text { Tieferer Älterer Deckenschotter } \\
\text { Mittlerer Älterer Deckenschotter }\end{array}$} & $\begin{array}{l}\text { Günz / Donau } \\
\text { Günz / Donau } \\
\text { Günz /Donau }\end{array}$ \\
\hline \multirow[t]{2}{*}{$\begin{array}{l}\text { Deckschotter } \\
\text { Unterer Deckschotter }\end{array}$} & & $\begin{array}{l}\text { Donau / Biber } \\
\text { Donau / Biber }\end{array}$ \\
\hline & \begin{tabular}{|l} 
Ältester Deckenschotter \\
Tieferer Ältester Deckenschotter \\
Höherer Ältester Deckenschotter
\end{tabular} & Biber \\
\hline Hochschotter & Hochschotter & Biber / Pliozän \\
\hline
\end{tabular}

* im Rot-Günz-Gebiet Unterer, Mittlerer und Oberer Zwischenterrassenschotter

System I nach GRAUL (1943, 1949, 1962) System II nach PENCK \& BRÜCKNER (1901-1909) und JERZ et al. (1975)

Ur-Argen angeliefert wurde. Östlich des RißIller-Gebiets anstehende kristallinreiche Schotter werden von EBerL (1930) in das Pliozän gestellt und als Ottobeurer Schotter bezeichnet. SINN (1972) sieht in ihnen unterpleistozäne, periglazial-fluviatile Schüttungen einer Ur-Eschach, -Rohrach und -Rottach, deren generelle Entwässerungsrichtung in nordöstlicher Orientierung verlaufen sein soll.

Die Literaturaufarbeitung dieses Absatzes verdeutlicht die bisherigen Schwierigkeiten bei der stratigraphischen Zuordnung der fluviatilen Ablagerungen im Raum südlich von Ulm und die Änderungen im Verlaufe der fortschreitenden Forschungstätigkeit. Die vorliegenden Untersuchungen sollen einen Beitrag liefern, die fraglichen Sedimente mit den Abfolgen des Illergletschergebietes zu korrelieren und eine lithostratigraphische Untergliederung anzubie- ten, die neue Erkenntnisse zur Geometrie und lithologischen Charakteristik der Paläotäler berücksichtigt. Dies erfolgt auch vor dem Hintergrund noch offener stratigraphischer Fragen, die nachfolgend skizziert werden und bislang die Korrelation von pleistozänen Abfolgen des nordwestlichen mit denen des nordöstlichen Alpenvorlandes erschwert haben

Das Riß-Iller-Gebiet liegt im Grenzbereich zweier derzeit gültiger stratigraphischer Gliederungen, welche insbesondere für den Zeitraum vor dem Holstein stark divergieren (Deutsche Stratigraphische Kommission 2002, LitT et al. 2005). Die für das badenwürttembergische Rheingletschergebiet erarbeitete Stratigraphie (Ellwanger et al. 1995, VILLINGER 2003) steht derjenigen für das bayerische Alpenvorland gegenüber (JERZ 1995, Doppler 2003). Eine aktuelle Übersicht 
unterschiedlicher Teilstratigraphien ist in НABBE (2003) wiedergegeben.

Die Diskrepanzen in den Stratigraphien westlich und östlich der Iller beruhen u. a. auf dem verschiedenen methodischen Ansatz, bei dem in Bayern die morphostratigraphische Untersuchung und in Baden-Württemberg die lithostratigraphische Interpretation von Beckenabfolgen im Vordergrund steht. Die besonders im Oberrheintal-Graben sowie im Hoßkircher Becken aufgefundenen Sedimente erlauben eine sequenz-stratigraphische Gliederung, mit der durch den Nachweis lithologischer Wechsel auf klimatische Veränderungen geschlossen werden kann. Die zeitliche Einordnung und die Korrelation mit anderen Quartärstratigraphien wird hier durch biostratigraphische und chronostratigraphische Informationen gestützt (LiTT et al. 2005). Die bayerische Quartärgliederung beruht auf der Gliederung von Schmelzwasserterrassen, welche im Gefolge der klassischen Untersuchungen von Penck \& BRÜCKNER (1901-1909) zum Nachweis immer komplexeren Terrassenabfolgen geführt hat. Mit der glazialen Serie wird für die letzten drei Glazialkomplexe (Mindel, Riss, Würm) die Verbindung von kaltklimatischem Klimasignal zum Sedimentkörper, der durch Gletscherschmelzwässer aufgeschüttet worden ist, hergestellt.

Den beiden stratigraphischen Konzepten gemeinsam ist die Zweiteilung in einen unterpleistozänen Abschnitt, in dem flächenhaft Deckenschotter verbreitet sind, und einen mittel- und oberpleistozänen Abschnitt. Dieser wird durch markante Moränensequenzen mit zugehörigen Terrassen geprägt. Von ElLWANGER et al. (1995) wird für den Rheingletscherbereich der Begriff morpho-tektonische Wende eingeführt und mit schubweisen Krustenbewegungen im Verlauf des Quartär in Verbindung gebracht, die solche auffallenden Fazieswechsel verursacht haben könnten. Die Bezeichnung morphologisch-tektonische Wende hat ScHLÜCHTER (1989) für das schweizerische Alpenvorland geprägt, um die älteren Deckenschotter-Vereisungen von den vier jüngeren Vorlandvereisungen gemäß des Systems von PENCK \& BRÜCKNER (1901-1909) zu unterscheiden.
Inkonsistenzen bei unterschiedlichen Regionalstratigraphien sind auch darauf zurückzuführen, dass die Dynamik der alpinen Gletscher regional unterschiedliche Überlieferungsdichten geologischer Zeugnisse aus den jeweiligen Zeitscheiben zur Folge hatten. Diese haben die Erosions- und Akkumulationstätigkeit im Vorland maßgeblich bestimmt, jedoch aufgrund ihrer differierenden Größe unterschiedlich auf Klimasignale reagiert. Der kleine Illergletscher hat beispielsweise schneller, aber wegen des kleineren Einzugsgebietes weniger ausgreifend auf eine Klimaänderung als der deutlich größere Rheingletscher reagiert. Dadurch sind Zeiten mit dominierender glazifluviatiler Akkumulation oder Erosion selbst bei zwei benachbarten alpinen Schmelzwassersystemen nicht zwangläufig zeitgleich erfolgt. Neben der sedimentologisch-petrographischen Untersuchung stellt daher die Rekonstruktion von Paläotälern aufgrund geometrischer Kriterien (Kap. 4) ein wichtiges Hilfsmittel zur Klärung der Ablagerungsreihenfolge dar.

\section{Methode}

Die quartären Ablagerungen des Untersuchungsgebiets wurden im Gelände geologisch kartiert und das Material in Aufschlüssen sedimentologisch-petrographisch aufgenommen. An Sedimentproben erfolgten im Labor weitere Untersuchungen, beispielsweise zur Korngrößenverteilung, Morphometrie, Schotterpetrographie und zum Schwermineralgehalt der Sandfraktion, um die Ablagerungsbedingungen sowie das Liefergebiet charakterisieren zu können. Es handelt sich dabei um Standardmethoden, die in der Literatur bereits mehrfach eingehend beschrieben worden sind (z. B. Löscher 1976, Schreiner 1992, BeckerHAUMANN 1995, 2005). Im Folgenden werden zwei Verfahren näher beschrieben, die bei der vorliegenden Untersuchung im Vordergrund standen und deren Ergebnisse in Kapitel 3 erläutert werden.

\section{Schotterpetrographie}

Um stratigraphische Zuordnungen unterstützen und die Liefergebiete der Schotter eingrenzen zu können, wurden geröllpetrographische 
Analysen an 43 Sedimentproben durchgeführt (Tab. 3). Pro Probe wurden durchschnittlich 300 Gerölle der Kornfraktion 20-63 mm (Grobkies) analysiert. Die Anzahl von 300 Geröllen erachten wir als ausreichend, denn Kontrollzählungen zeigten, dass bei 300, 400, 500 und 600 Komponenten die prozentuale Zusammensetzung innerhalb des statistischen Fehlers gleich bleibt. Fezer (1969) hat für das Untersuchungsgebiet beschrieben, wie die petrographische Zusammensetzung von der Korngröße beeinflusst wird, so dass an Stichproben ebenso die Fraktion 11,2-20 mm (Fein-, Mittetlkies) untersucht wurde. Die Zählergebnisse dieser Fraktion stimmen mit denen der Grobkiesfraktion überein. Folgende Gesteinstypen wurden unterschieden und zu fünf lithologischen Gruppen zusammengefasst:

\section{Kalkstein}

Kalkstein - Meist mikritische alpine Kalke, bei denen schwarze Kalke, helle, grauweiße Kalke, rotbraune Kalke, gelbe Fleckenkalke gesondert ausgezählt wurden. Kalke mit gelben Krusten entstehen durch Verwitterung aus mikritischen Kalken.

Kieselskelette - Völlig entkalkte, splittrig zerfallende Residuate unreiner Kalksteine. Nur das Kieselgerüst ist erhalten.

\section{Dolomit}

Dolomit in unterschiedlichster Erhaltung von leicht verwitterten Geröllen bis zu stark sandenden, leicht zerfallenden so genannten Dolomitaschen (FEZER 1969).

\section{Sandstein und Konglomerat}

Sandstein - Fein- bis mittelkörnige Sandsteine und Konglomerate, z. T. stark karbonatisch mit Unterteilung in helle Molassesandsteine, Flyschsandsteine, roter Sandsteine, Quarzsandsteine und Glaukonitsandsteine.

\section{Kristallin}

Kristalline - Meist helle Gneise und dunkle Amphibolite sowie Epidot-Chlorit-(Glimmer-) Schiefer und hellgrün-weiß gebänderte Epidotquarzite. Selten kommen rote Granite vor. Die Gneise zerfallen durch Verwitterung meist leicht.

\section{Harte}

Gangquarz - Oft grobe, milchig trübe, weiße bis gelbliche Gerölle.

Quarzit - Quarzite metamorpher Abkunft und kieselig gebundene Sandsteine aller Farbvariationen.

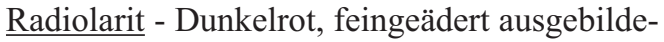
te Kieselgesteine.

Hornstein - Schwarzbraune Kieselkonkretionen, die nur selten $(<1 \%)$ vorkommen und mit den Radiolariten zusammengefasst wurden.

\section{Schwermineralanalysen}

Nach den Untersuchungen von WeYL (1952) wurden im Riß-Iller-Gebiet erneut systematische Schwermineralanalysen durchgeführt, um ergänzende Informationen über die Herkunft und Transportrichtung der Sedimente zu erhalten (Tab. 4). Dazu wurde an 97 Proben die Korngrößenfraktion $0,4-0,063 \mathrm{~mm}$ abgesiebt und 15 Minuten lang mit 25\%-iger Salzsäure gekocht. Dadurch werden Eisenhydroxidverkrustungen beseitigt, die die spätere Analyse behindern, jedoch wird auch das Mineral Apatit entfernt. Die Schweretrennung erfolgte mit Natrium-Polywolframat $\left(3 \mathrm{Na}_{2} \mathrm{WO}_{4} \cdot 9 \mathrm{WO}_{3}\right.$ $\cdot \mathrm{H}_{2} \mathrm{O}$ ) mit einer Dichte von $2,85 \mathrm{~g} / \mathrm{cm}^{3}$ in einer Zentrifuge bei $2500 \mathrm{U} / \mathrm{min}$. Nach Reinigung und Trocknung wurden die Schwerminerale in Kanadabalsam mit einem Brechungsindex von $\mathrm{n}=1,54$ eingebettet. Nach anschließender Aushärtung und Reinigung der Präparate erfolgte die polarisationsoptische Bestimmung von rund 100 transparenten Schwermineralkörnen nach BoenigK (1983).

$\mathrm{Zu}$ Auswertungszwecken sind die Minerale gemäß ihrer Verwitterungsresistenz nach BoENIGK (1983) in folgende Gruppen zusammengefasst worden:

I Instabile - Granat, grüne und braune Hornblende, Klinopyroxen

II Stabile - Epidot, Staurolith

III Sehr Stabile - Andalusit, Disthen, Sillimanit IV Extrem Stabile - Zirkon, Rutil, Turmalin. 


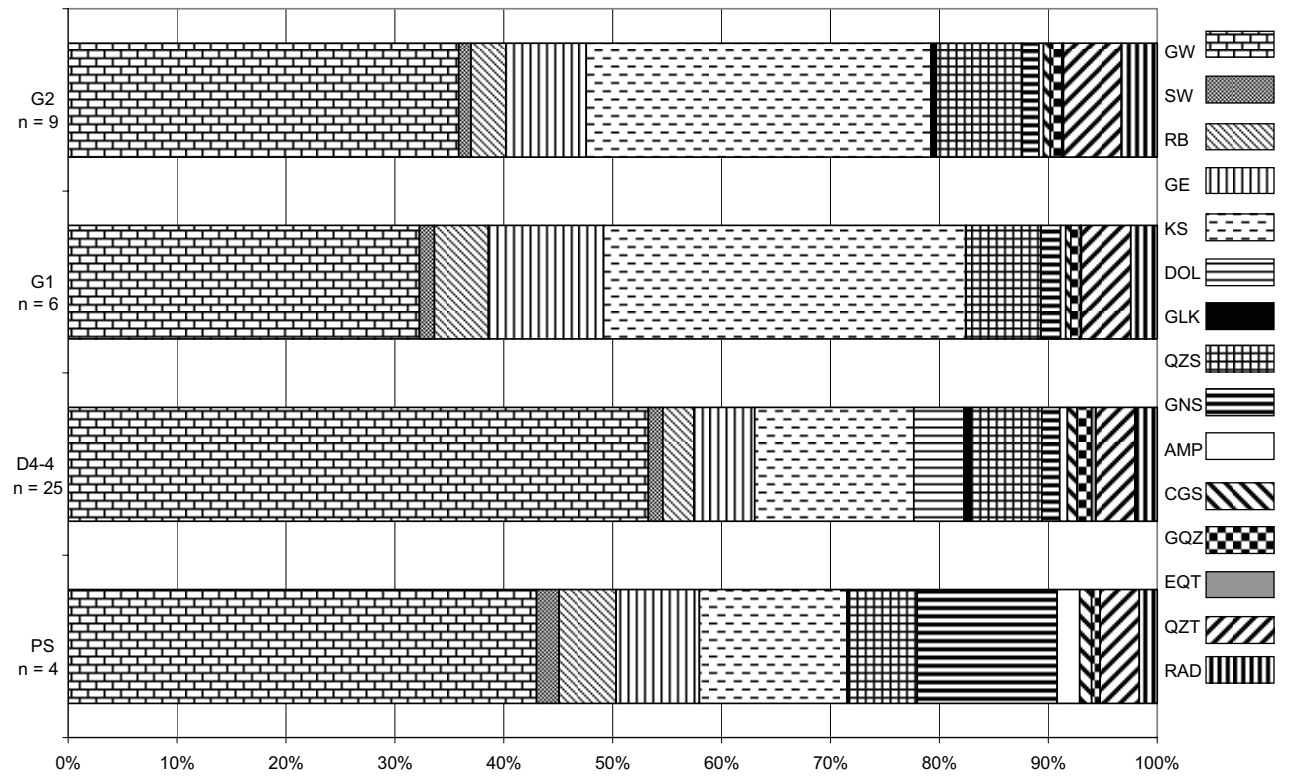

Abb. 2: Mittelwerte der schotterpetrographischen Analysen. Es bedeuten G2: Günz-2-zeitlicher Schotter, G1: Günz-1-zeitlicher Schotter, D4-4: Donau-4-4-zeitlicher Schotter, PS: Ältester Periglazialschotter, Abkürzungen in der Legende wie in Tabelle 2.

Fig. 2: Mean values of the petrography of the gravels. G2: Günz-2-age gravels, G1: Günz-1-age gravels, D44: Donau-4-4- age gravels, PS: Ältester Periglazialschotter, abbreviations of the petrography see table 2.

\section{Ergebnisse}

\section{Schotterpetrographie}

Aufgrund des hohen Alters der Sedimente und der feuchten Witterungsbedingungen der Region (heutiger Wert: 800-900 mm/Jahr) spielt die Verwitterung bei der schotterpetrographischen Zusammensetzung eine große Rolle. Bei geringmächtigen Schottern kann es dadurch über das gesamte Profil zur Anreicherung von harten Komponenten (Quarz, Quarzit, Hornstein, Radiolarit) kommen, während karbonatische Gerölle (Kalkstein, Dolomit) stark korrodiert oder aufgelöst sein können.

Über den Anteil an Kieselskeletten kann nur näherungsweise auf den primären Gehalt von Karbonaten geschlossen werden. Berechnungen des ursprünglichen Anteils von Karbonatgeröllen, wie sie VILLINGER (2003) für die Sedimente der Restschotter im Raum Ehingen-Rottenacker durchführt, erscheinen problematisch, da der Anteil völlig aufgelöster Karbonate nicht in die Kalkulation eingeht. Zudem können laterale Schwankungen der Schotterzusammensetzung zu Fehlrechnungen führen, wenn Daten aus anderen Bereichen eines Schotterniveaus als Referenz herangezogen werden. Aus dem erweiterten Untersuchungsbereich gibt es zahlreiche Beispiele für lateral heterogene Zusammensetzungen von Schotterkörpern (BECKER-HAUMANN 2005).

Zuverlässig gelingt die Unterscheidung des Ältesten Periglazialschotters (Bezeichnung nach DOPPLER 2003, kristalline Liegendfazies nach SINN 1972) auf den Schotterfeldern von Ochsenhausen, Erolzheim sowie Kellmünz von den überlagernden glazifluviatilen Schottern (Hangendfazies nach LÖSCHER 1976), die dem Donau 4-4 zugerechnet werden (Abb. 2). Während die Kristallingehalte der glazifluviatilen Schotter zwischen $1 \%$ und $7 \%$ liegen, weisen die Ältesten Periglazialschotter solche von 1319\% auf. Diese Unterschiede lassen sich nicht auf Verwitterungseffekte zurückführen, da die 
Anteile an Kieselskeletten annähernd gleich sind und bei den Proben von der Ochsenhausener und der Erolzheimer Hochfläche sogar unter denen der Hangendfazies liegen. Das Spektrum der Kristallingerölle wird in den Sedimenten der Ältesten Periglazialschotter im Wesentlichen von hellen Gneisen und dunklen Amphiboliten geprägt, daneben finden sich EpidotChloritschiefer mit 2-3\%. Der Anteil an Epidot-Chloritschiefern beträgt in der glazifluviatilen Hangendfazies demgegenüber nicht mehr als 2\%. Der von Geiger (1969) und LÖSCHER (1976) angeführte hohe Anteil heller Gneise aus dem Silvretta-Gebiet wird durch die eigenen Analysen bestätigt.

Die Untersuchungen innerhalb des Donau-4Niveaus zeigen, dass das Kristallinspektrum weitaus differenzierter ist, als von GEIGER (1969), Eichler (1970) und LÖSCHER (1976) beschrieben. Neben den in Tabelle 3 angegebenen Gesteinsarten treten Diabase, rote Granite und dunkelgrüne Epidotquarzite auf. Vereinzelt finden sich rote Granite mit bis zu $14 \mathrm{~cm}$ Durchmesser, wie sie in den jüngeren, mindelzeitlich eingestuften Schottern als typisches Rheingletschermaterial beschrieben werden (Geiger 1969, HaAg 1982, Schaefer 1995). Die qualitativen Untersuchungen von Becker-Haumann (1996) zeigten bereits, dass diese Komponenten ebenso in pleistozänen Illerschottern verbreitet sind. Nach SinN (1972) und Löscher (1976) ist das Auftreten der genannten Kristallingesteine auf Umlagerung aus dem tertiären Hochgratfächer (Eschach-Kürnacher Wald) zurückzuführen.

In den Sedimenten des Hochgratfächers treten bevorzugt helle Muskowit-Gneise (EBERHARD 1987, HALDER 1988) und Amphibolite (EBERHARD 1987, HANSEL 1989) auf, wobei die Amphibolite bereits im Adelegg-Gebiet starke Verwitterungsspuren aufweisen, da sich dort die Gerölle bereits auf sekundärer Lagerstätte befinden. Sie entstammen dem Silvretta-Kristallin (TANNER 1944). Im Zuge der pleistozänen Umlagerung wurden die Gehalte der Amphibolitgerölle weiter reduziert, so dass heute nur Werte von $1-3 \%$ in den Schmelzwasserschottern erreicht werden (Tab. 3, GeIGer 1969, LÖSCHER 1976). Die Ausgangsgehalte von Amphiboliten und Gneisen sind im Hochgrat- fächer mit 1-20\% recht variabel (HALDER 1988, EBERHARD 1986). Das Geröllspektrum wird bei den Schottern der glazifluviatilen Hangendfazies (Donau 4) von Gesteinen der SilvrettaDecke (Kristallin), der osthelvetischen Decken und aus dem Ostalpin (Sedimente) dominiert (Geiger 1969, Villinger 2003).

Die geröllpetrographischen Untersuchungen im Arbeitsgebiet (Abb. 2) zeigen, dass es innerhalb der untersuchten Einheiten - der Ältesten Periglazialschotter sowie der glazifluviatilen Fazies aus der Donau- und Günzeiszeit - zu einer nur unwesentlichen Vergrößerung des alpinen Einzugsgebietes von Rhein- und Illergletscher kam. Wesentlich ist, dass das periglazial-fluviatile Material große Anteile von Adelegg-Material (hoher Silvretta-Anteil, geringer Amphibolitgehalt), die glazifluviatilen Schotter hingegen überwiegend Komponenten der Ostalpinen Decken (geringer Silvretta-Anteil in Bezug zum Amphibolitgehalt, erhöhter Chloritschiefer-Gehalt) enthalten. Eine Unterscheidung zwischen donau- und günzzeitlichen Terrassenschottern ist nicht möglich. Die unterpleistozänen Sedimente zeigen insgesamt eine hohe Diversität bei den Kristallingesteinen.

Eine petrographische Differenzierung der glazifluviatilen Ablagerungen gelingt erst bei den haslach- und mindelzeitlichen Schottern (Graul 1953, Geiger 1969, HaAg 1982, SchaeFER 1995), denn das zentralalpine Grundgebirge wurde durch glazigenen Einfluss zunehmend abgetragen, was zur lithologischen Veränderung des Liefergebiets führte (VILLINGER 2003). Eine weitere Auffälligkeit der Hangendfazies westlich des heutigen Illertals ist das weitgehende Fehlen des Dolomits (0-1\%). Nach DOPPLER (2003) zeigen auch die eigenen Untersuchungen, dass dort nur noch geringe Reste nicht entkalkter Schotter erhalten geblieben sind. Dies könnte für einen Verwitterungseffekt sprechen, jedoch sind die Schotter östlich des Illertales von vergleichbarem Alter und führen Dolomit in Anteilen von 15 bis 22\% (EBERL 1930, Eichler 1970, RöGner 1986, Sinn 1972). Dolomite treten verstärkt in haslach- und mindelzeitlichen Sedimenten auf (SCHREINER \& Ebel 1981, HaAg 1982, Schaefer 1995).

Für die höheren Dolomitgehalte des Kellmünzer Schotters ist weniger der Einfluss des Iller- 

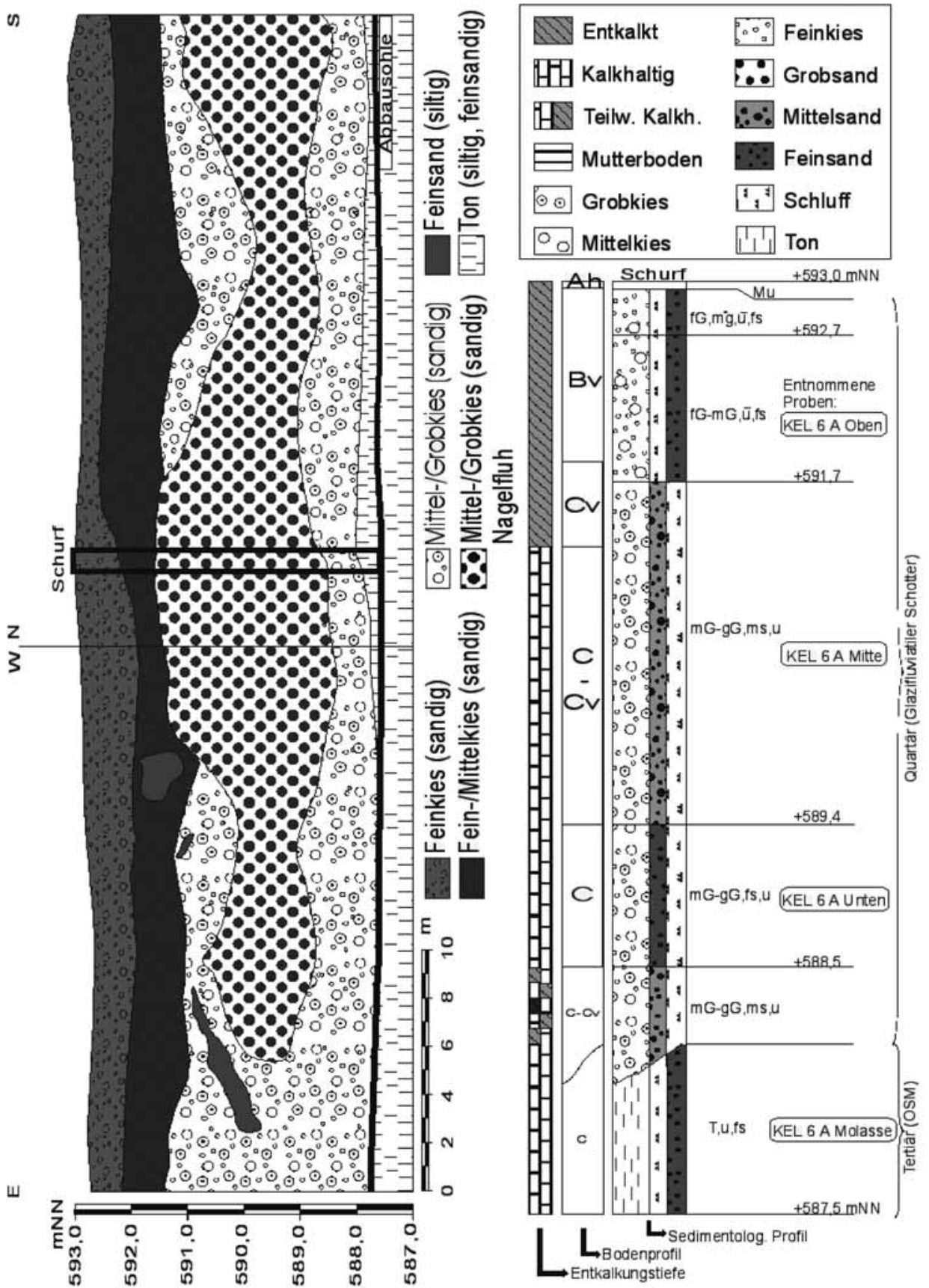

Abb. 3: Kiesgrube bei Untereichen. Links: Skizze der Abbauwand im östlichen Abbaubereich. Rechts: Profil des Schurfes. Die Höhe des Schurfes ist nicht maßstäblich, sondern in Teilbereichen aufgespreizt.

Fig. 3: Gravelpit near Untereichen. The sketch on the left displays the eastern cut of the pit. On the right the section of the deposits is shown. The vertical scale is exaggerated. 


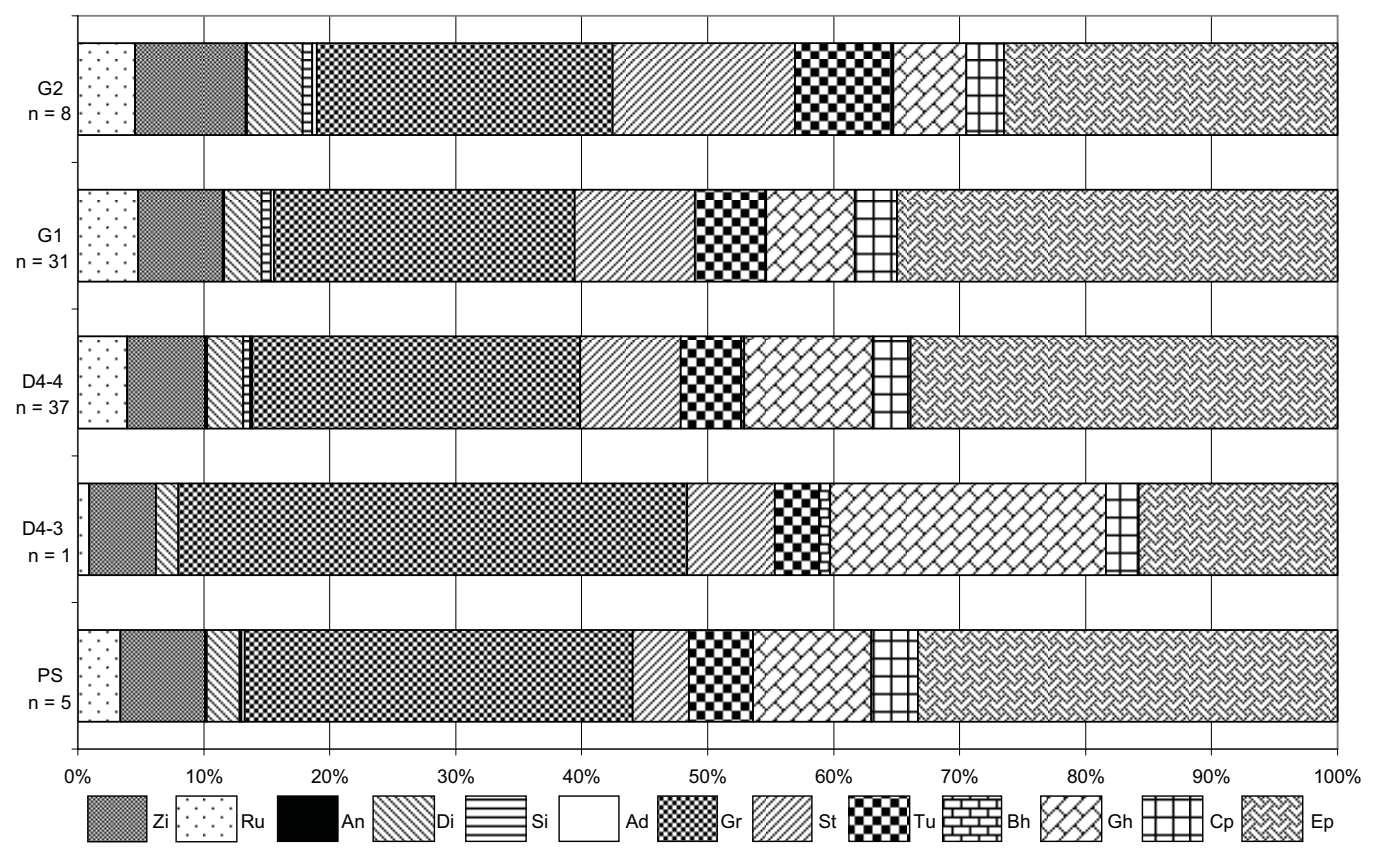

Abb. 4: Mittelwerte der Schwermineralanalysen der untersuchten Schotter. Es bedeuten G2: Günz-2-zeitlicher Schotter, G1: Günz-1-zeitlicher Schotter, D4-4: Donau-4-4-zeitlicher Schotter, PS: Ältester Periglazialschotter, Abkürzungen in der Legende wie in Tabelle 3.

Fig. 4: Mean values of the heavy mineral analyses. G2: Günz-2-age gravels, G1: Günz-1-age gravels, D4-4: Donau-4-4- age gravels, PS: Ältester Periglazialschotter, abbreviations of the heavy minerals see table 3.

gletschers, als die lokal reduzierte Verwitterungswirkung verantwortlich. Das Material des Kellmünzer Schotters, das bei Untereichen untersucht wurde, ist vermutlich vom Rhein- und nicht vom Illergletscher (SINN 1972) geliefert worden. Dafür sprechen die Einregelungsmessungen mit einer Vorzugsrichtung aus $212^{\circ}$. Es ist allerdings darauf hinzuweisen, dass sich die Herkunft dieser unterpleistozänen Schotter derzeit nicht zweifelsfrei klären lässt, da die Petrographie keine eindeutigen Schlüsse zulässt und die Dichte der Paläoströmungsinformationen vergleichsweise gering ist.

Der Schotter bei Untereichen (Abb. 3) besteht im unteren Bereich weitgehend aus schluffigen bis feinsandigen Grobkiesen, die mit korngestützten, mittelsandigen Grob- bis Mittelkiesen wechseln. Darüber folgen mittelsandige Grob- bis Mittelkiese in Wechsellagerung mit grobsandigen Grobkiesen. Diese gehen zum Hangenden in z. T. stark schluffige Fein- bis
Mittelkiese und mittel- bis feinsandige Grobkiese mit vereinzelten Blocklagen über. Die Entkalkungstiefe der Abfolge beträgt etwa $2 \mathrm{~m}$, darunter folgt in unregelmäßiger Ausprägung - auf zirkulierende Porenwässer und sekundäre Kalkausfällung zurückgehend - zu Nagelfluh verbackener Schotter. Ein stellenweise direkt über der Molasse auftretender unterer Entkalkungshorizont in den Schottern ist wahrscheinlich auf Prozesse an der Oberfläche des Grundwassers zurückzuführen. Aufgrund der Überprägung der Sedimente durch Verwitterung (Braunerde-Bodenbildung) und Porenwasser steht lediglich etwa ein Meter eines nicht oder kaum alterierten Schotterrestes an (C-Horizont des Bodenprofils in Abb. 3). Insbesondere die Ausbildung bzw. Erhaltung des Sedimentes im oberen, stärker den Verwitterungsprozessen ausgesetzten Schotterbereich ist mit den Verhältnissen der anderen untersuchten Schottervokommen vergleichbar. 

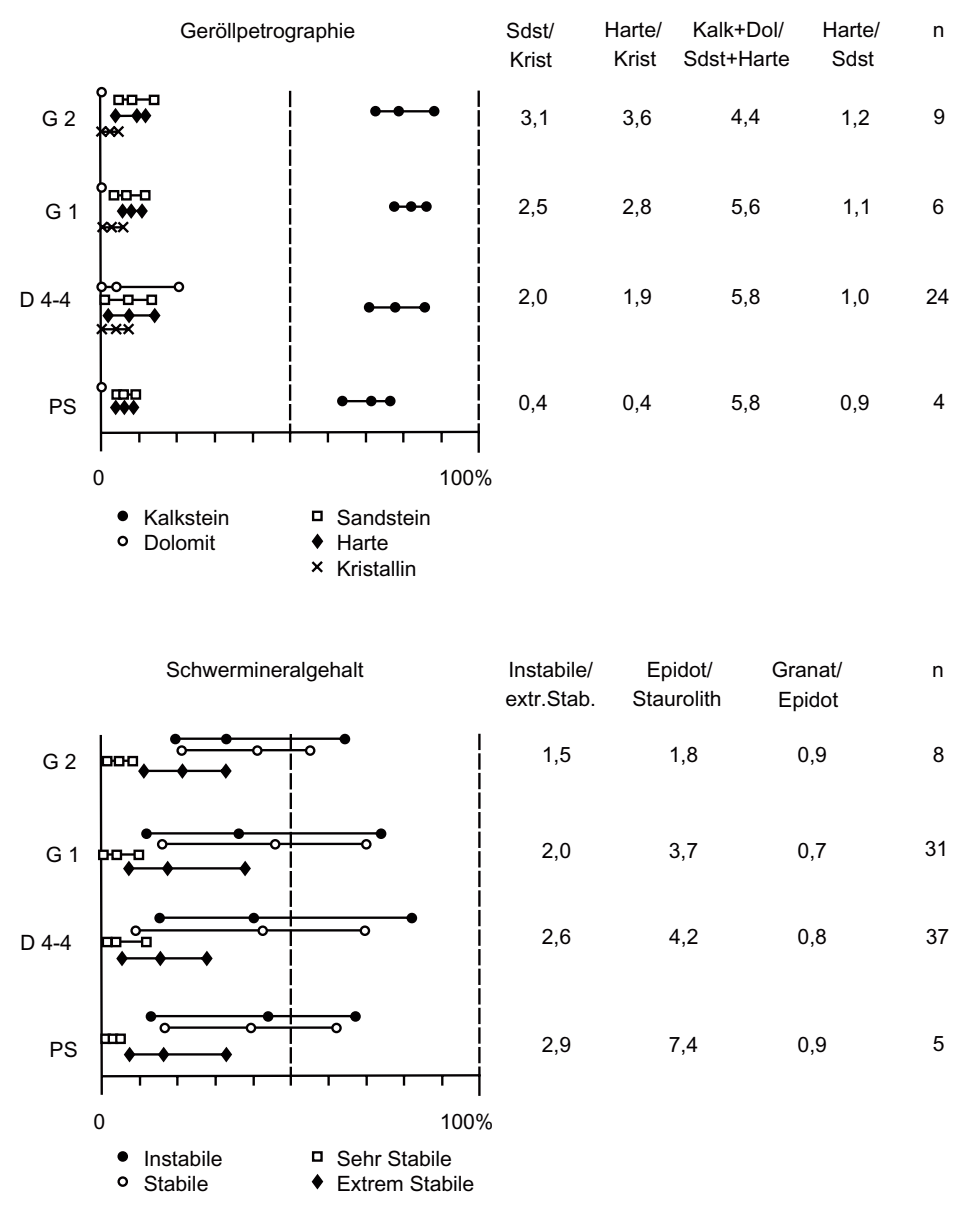

Abb. 5: Auswertung der Analysenergebnisse durch Quotientenbildung einzelner Geröll- (oben) bzw. Schwermineralgruppen (unten). n: ausgewertete Probenzahl.

Oben links sind die Minimal-, Mittel- und Maximalgehalte von fünf petrographischen Gruppen gemäß Tabelle 2 eingetragen. Daran ist die Streuung der Ergebnisse innerhalb der stratigraphischen Einheiten PS (Ältester Periglazialschotter), D 4-4 (Donau-4-4-zeitlicher Schotter), G 1 (Günz-1-zeitlicher Schotter) und G 2 (Günz-2-zeitlicher Schotter) zu erkennen. Auf der rechten Seite sind Quotienten aus den Mittelwerten der angegebenen Geröllgruppen errechnet, die eine systematische Veränderung von den ältesten zu den jüngeren Terrassen erkennen lassen. Im unteren Teil der Abbildung ist die Auswertung für die Schwermineralgehalte in den untersuchten Terrassen angegeben. Daraus lässt sich schließen, dass über die Zeit eine Veränderung der Sedimentanlieferung erfolgt ist (weitere Erläuterung im Text).

Fig. 5: Petrographic (above) and heavy mineral analyses (below) of the investigated gravels.

The minimal, mean, and maximum values of petrographic composition of the gravels (tab. 2) are shown above left. The high variation of the stratigraphic units PS (Ältester Periglazialschiotter), D 4-4 (Donau-4-4age gravels), G1 (Günz-1-age gravels) and G2 (Günz-2-age gravels) can be recognized. On the above right diagram the quotients of the mean values for several petrographic associations are given showing a systematic trend from the older towards the younger terrace units. In the lower part of the figure the analogous calculations of the heavy mineral content is shown (tab. 3). This reveals the conclusion that the sediment supply has changed over time. For further explanations see text. 
In Übereinstimmung mit DOPPLER (2003) ergibt sich, dass eine schotter-petrographische Unterscheidung nur zwischen Ältestem Periglazialschotter und den glazifluviatilen Schottern der Hangendfazies möglich ist. Das Fehlen von Leitgeröllen verhindert eine klare Unterscheidung zwischen Material, das durch den Rhein bzw. die Iller angeliefert worden ist. Trotz der generellen verwitterungsbedingten Einschränkung bei der Interpretation des Dolomitgehaltes ist auffallend, dass die glazifluviatilen Vorkommen westlich des heutigen Illertales weitgehend dolomitfrei, die östlich anschließenden dolomitführend sind. Werden die untersuchten Geröllfraktionen in petrographische Gruppen gemäß der Tabelle 3 zusammengefasst, zeigt sich, dass der durchschnittliche Gehalt an Kalksteinen zu den jüngeren Terrassenschottern von $71,5 \%$ (Ältester Periglazialschotter) auf etwa $80 \%$ (Günz 2) zunimmt (Abb. 5). Werden die mittleren Anteile der Sandsteine und der Harten (Radiolarit, Hornstein, Quarz, Quarzit) in Relation zu den Kristallinen gesetzt, ergibt sich bei den donau- und günzzeitlichen Terrassen ein klarer Trend zu höheren Werten bei den günzzeitlichen Ablagerungen, der auf eine relative Abreicherung kristalliner Gerölle zurückgeht (Abb. 5). Bei den Ältesten Periglazialschottern liegen die Werte aufgrund der hohen Kristallinanteile von durchschnittlich 16\% bei 0,4. Werden die Karbonate (Kalkstein und Dolomit) ins Verhältnis zu den Sandsteinen und Harten gesetzt, sinken die Quotienten von 5,8 bei den Ältesten Periglazialschottern auf 4,4 bei den Günz 2-Sedimenten ab. Ein Trend zu höheren Werten ist bei dem Anteil der Harten zu dem der Sandsteine von den alten zu den jungen untersuchten Terrassenschottern ablesbar, was eine starke Zunahme der Harten um etwa 4 Prozentpunkte entspricht.

\section{Schwerminerale}

Das Schwermineralspektrum wird bei den untersuchten Ablagerungen von der charakteristischen alpinen Schwermineralassoziation von Granat, Epidot und Staurolith dominiert und entspricht den von Löscher et al. (1978) und RÖGNER (1986) mitgeteilten Werten. Im Ältesten Periglazialschotter betragen die Gehalte von Granat 38-58\%, für Epidot 14-59\% und für Staurolith 2-9\% (Proben SW-12, 78, 84 gemäß Tab. 4). Grüne Hornblende liegt mit maximal $20 \%$ vor (Probe SW-84 gemäß Tab. 4), jedoch werden $6 \%$ meist nicht überschritten (Abb. 4, Tab. 4). Diese Werte bestätigen die Daten, die LÖSCHER et al. (1978) für den Aufschluss Untereichen mitgeteilt haben.

Die große Streubreite bei den einzelnen Mineralen sowie bei den aufgrund unterschiedlicher Verwitterungsresistenz gebildeten Mineralgruppen (Instabile, Stabile, sehr Stabile, extrem Stabile) lässt keine sichere Unterscheidung der Terrassensedimente zu. Dennoch lassen sich einige Tendenzen aus den Daten ableiten. Im Mittel ist von den D4-4- zu den G2-Schottern eine Abnahme des Gehaltes an grüner Hornblende sowie eine Zunahme der Staurolith- und Turmalinanteile zu verzeichnen (Abb. 4, Tab. 4). Das Verhältnis von Epidot zu Staurolith sinkt innerhalb der vier Einheiten von 7,4 auf 1,8. Da beide Minerale ähnlich verwitterungsresistent sind, zeigt dies eine primäre Zunahme des Stauroliths an. Trotz der Schwankungsbreiten ist auffallend, dass der mittlere Gehalt von Granat wie auch der ganzen Gruppe der instabilen Schwerminerale (Granat, Hornblende, Klinopyroxen) von den Ältesten Periglazialschottern zur Günz 2-Terrasse um etwa 11 Prozentpunkte abnimmt. Gleichzeitig steigt der Gehalt der extrem stabilen Schwerminerale um etwa 6 Prozentpunkte an. Dies spiegelt sich in einer Verringerung des Quotienten aus dem Anteil instabiler und extrem stabiler Schwerminerale von 2,9 auf 1,5 zu den jüngeren Einheiten wider (Abb. 5). Die Befunde deuten insgesamt darauf hin, dass über die Zeit eine Veränderung des Liefergebiets stattgefunden hat, in deren Verlauf der Granatgehalt abnahm und der Staurolithanteil stieg. Die Sedimentschüttung ist ferner dadurch ausgezeichnet, dass der Epidotgehalt den des Granat um bis zu einem Drittel übersteigt.

\section{Flussgeschichtliche Interpretation der Befunde}

Älteste Periglazialschotter sind auf dem Erlenmooser Riedel, Erolzheimer Riedel, Kellmünzer Feld und Teilen des Eisenburger Feldes 


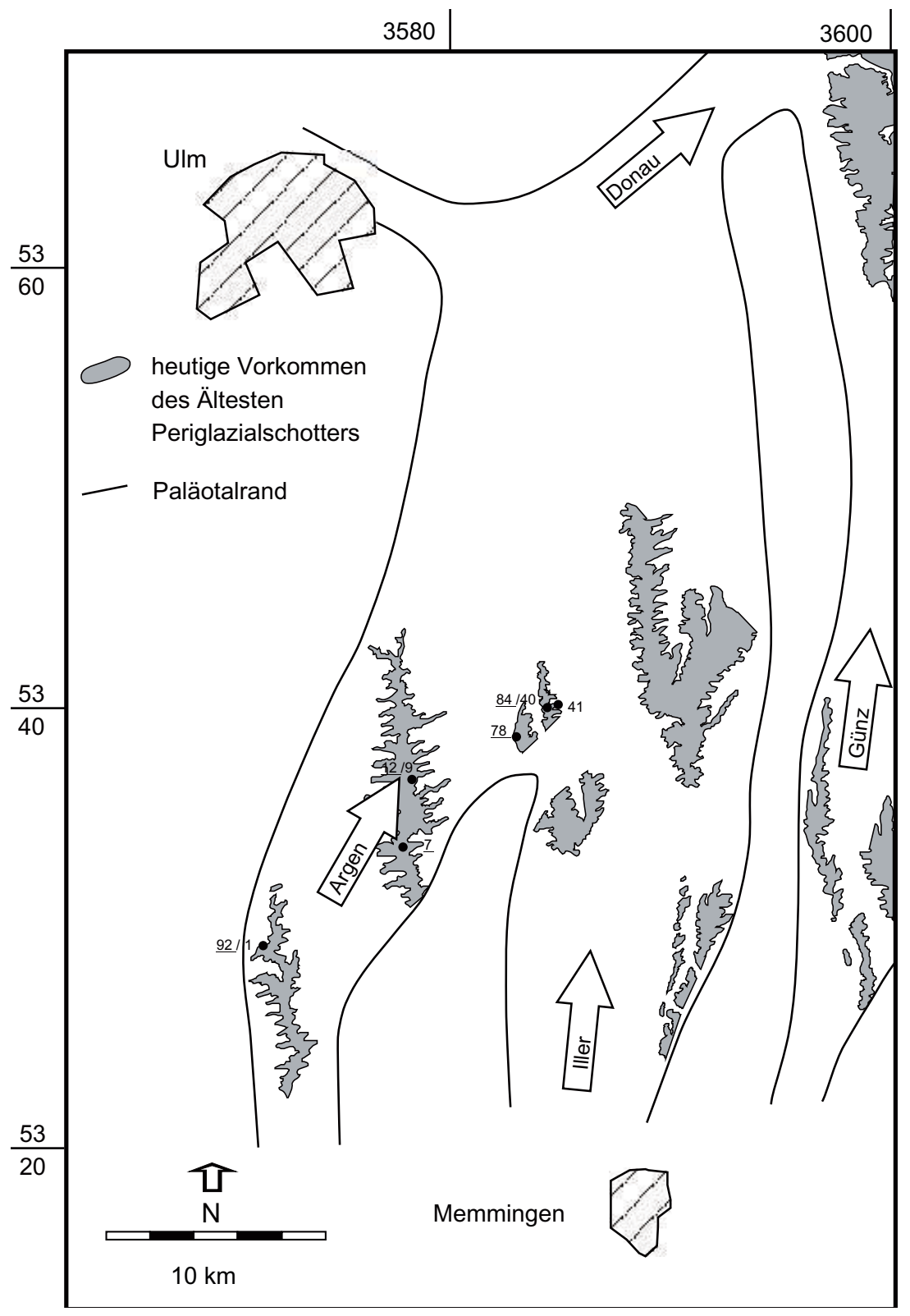

Abb. 6: Talverlauf zur Zeit der Ablagerung der Ältesten Periglazialschotter (Donau 2). Die Ziffern geben die Probenentnahmestellen für petrographische Analysen (ohne Unterstrich, Nummerierung nach Tab. 2) und Schwermineraluntersuchungen (mit Unterstrich, Nummerierung nach Tab. 3) an.

Fig. 6: Valley during Donau 2 (Ältester Periglazialschotter). The sample locations are indicated by underlined numbers for petrographic analyses (tab. 2) and numbers for heavy mineral analyses (tab. 3 ). 


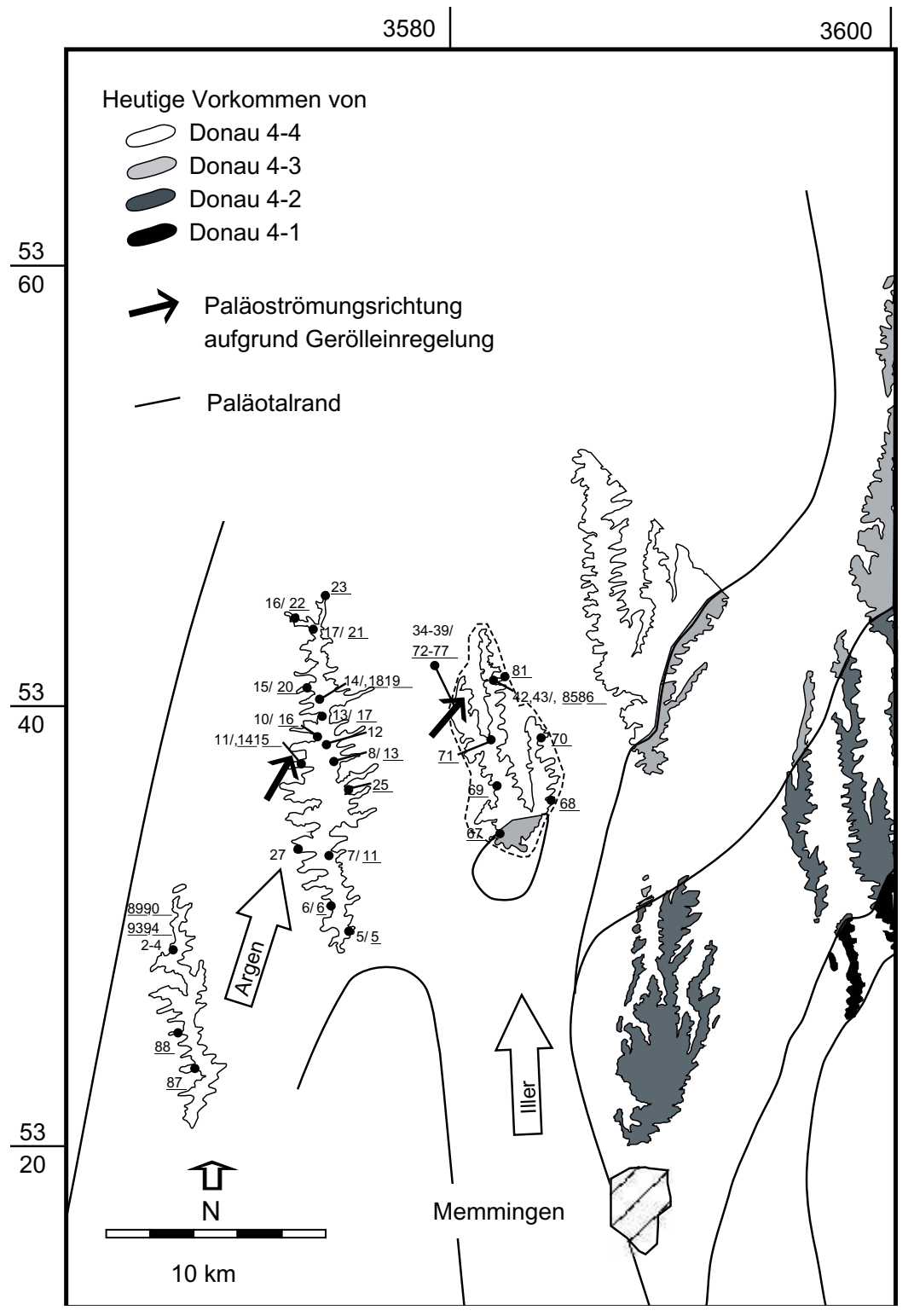

Abb. 7: Talverlauf zur Zeit der Ablagerung von Donau 4-1 bis Donau 4-4. Die Ziffern geben die Probenentnahmestellen für petrographische Analysen (ohne Unterstrich, Nummerierung nach Tab. 2) und Schwermineraluntersuchungen (mit Unterstrich, Nummerierung nach Tab. 3) an. Donau 4-1 und Donau 4-2 nach Becker-Haumann (2005).

Fig. 7: Valley from Donau 4-1 to Donau 4-4. Indications see fig. 6. Donau 4-1 and Donau 4-2 after BECKERHAUMANN (2005). The sample locations are indicated by underlined numbers for petrographic analyses (tab. 2) and numbers for heavy mineral analyses (tab. 3). 
sowie des Inneberg-Reisensburger Feldes erhalten (Abb. 6). Die aus südwestlicher bzw. südlicher Richtung strömenden Wässer von Argen und Iller vereinigten sich nahe des Ortes Kellmünz zu einem nordwärts gerichteten, etwa $14 \mathrm{~km}$ breiten Tal. Dieses wurde bereits in der vorausgegangenen Donau-Eiszeit angelegt und von den periglazialen Bächen weiterhin genutzt (Becker-Haumann \& Gerth im Druck). Das Gefälle des Illertales nimmt von 3\%o auf 2,3\%o ab, das des Argentals ist mit 4,2\%o etwas steiler. Aufgrund einer abweichenden Entwicklung der Einfallswerte ist das Günztal isoliert vom Illertal nordwärts verlaufen und bei Offingen in die Donau gemündet. Wie 3D-Modellierungen gezeigt haben (Becker-Haumann \& Gerth im Druck) ist der Älteste Periglazialschotter mit dem Donau-zeitlichen D2-Niveau im Lechtal zu korrelieren. Die Gefällswerte der Donau, die relativen Altersbezüge zu den überlagernden glazifluviatilen Sedimenten des Ältesten Periglazialschotters finden ebenso wie die Terrassenabfolgen des Illergletscher-Vorlandes bei dieser Einstufung Berücksichtigung.

Die glazifluviatile Hangendfazies der Ältesten Periglazialschotter wird in BECKER-HAUMANN (2005) in vier Einheiten gegliedert, die eine Verlagerung des Illertales nach Nordwesten dokumentieren. Die ältesten Ablagerungen des Niveaus Donau 4-1 sind kristallinarm und dolomitreich (Löscher 1976). Die Schotterpetrographie und schwermineralogische Zusammensetzung der in vorliegender Arbeit beprobten Einheit Donau 4-4 ist lateral einheitlich und durch geringe Dolomitgehalte $(<1 \%)$ und Kristallingehalte um 5\% charakterisiert. Abbildung 7 zeigt die in nordwestlicher Richtung jünger werdenden Ablagerungen und die Ränder der Paläotäler im erweiterten IllerRiß-Gebiet. Die ältesten Schüttungen Donau 4-1 bis Donau 4-3 sind im Gebiet nördlich und östlich von Memmingen verbreitet und bauen den flächenmäßig dominierenden Teil des Illergletscher-Vorlandes auf. Die jüngste Einheit Donau 4-4 tritt hingegen im Iller-Riß-Gebiet auf, wo sie den Erlenmooser und Erolzheimer Riedel, weite Bereiche des Kellmünzer sowie den südlichen Teil des Weißenhorn-Kissendorfer Feldes (Bucher Schotter) einnimmt.

Problematisch und in Becker-Haumann (2005) diskutiert ist das Fehlen im Gelände auskartierbarer Talränder und Uferzonen an den Grenzen der einzelnen Niveaus, was sich allerdings durch eine kontinuierliche Talverlagerung gemäß der Vorstellungen von BECKER-HAUMANN (1999) erklären lässt. Die in BECKER-HAUMANN \& GERTH (im Druck) aufgeführten Daten zur Talgeometrie zeigen, dass sich bei der Annahme von vier Einheiten plausible Werte zur primären Mächtigkeit der Talfüllung und zum Gefälle des Terrassenkörpers ergeben. Ebenso stehen die Geländebefunde zur Paläoströmungsrichtung und den erhaltenen Schottermächtigkeiten im Einklang mit dem sich ergebenden Talverlauf. Die Grenzen, die in Abbildung 7 eingezeichnet sind, stellen daher Resultate dieser 3D-Modellierungen und keine Kartierergebnisse dar. Für das Iller-RißGebiet resultiert ein Tal, das während des Donau 4-4 von Ochsenhausen nach Nordnordosten verlief (Argental) und den östlichen Rheingletscher entwässerte (Abb. 7). Es erhielt einen Zufluss vom Illergletscher aus dem Gebiet westlich von Memmingen (Illertal).

Die zwei jüngsten untersuchten Schotterniveaus werden in Übereinstimmung mit DOPPLER (2003) in die Günzeiszeit gestellt. Die Günz 1-zeitliche Terrasse ist auf dem Sendener Feld und dem östlichen Bereich des Holzstöcke-Feldes erhalten (Abb. 8). Der Talverlauf ist hier - im Bereich zwischen Dietenheim und Ulm - sicher rekonstruierbar, während die Informationen weiter südlich für eine paläogeographische Aussage zu spärlich sind. Einzig der Südsporn des Holzstöcke-Feldes besteht östlich des Ortes Schwendi aus korrelaten Ablagerungen, die einen Zufluss der Argen aus dem östlichen Rheingletschergebiet anzeigen. Der Verlauf der Iller ist für diese Zeit weithin unsicher. Der Zusammenfluss von Iller und Argen kann für das Günz 1 im Bereich Dietenheim (im heutigen Iller-Tal) und für das Günz 2 im Bereich von Gutenzell (im heutigen Rot-Tal) vermutet werden (Abb. 8 und BECKERHaUmann \& Gerth im Druck). Die Ergebnisse der 3D-Modellierungen zeigen, dass sich der Wortelstettener Schotter am Nordsporn der Zusamplatte sowie einige Vorkommen auf der Aindlinger Terrassentreppe mit den Ablagerungen des Argentales mit einem Gefälle von ca. $0,8 \%$ verknüpfen lassen (BECKER-HAUMANN \& GERTH im Druck). 


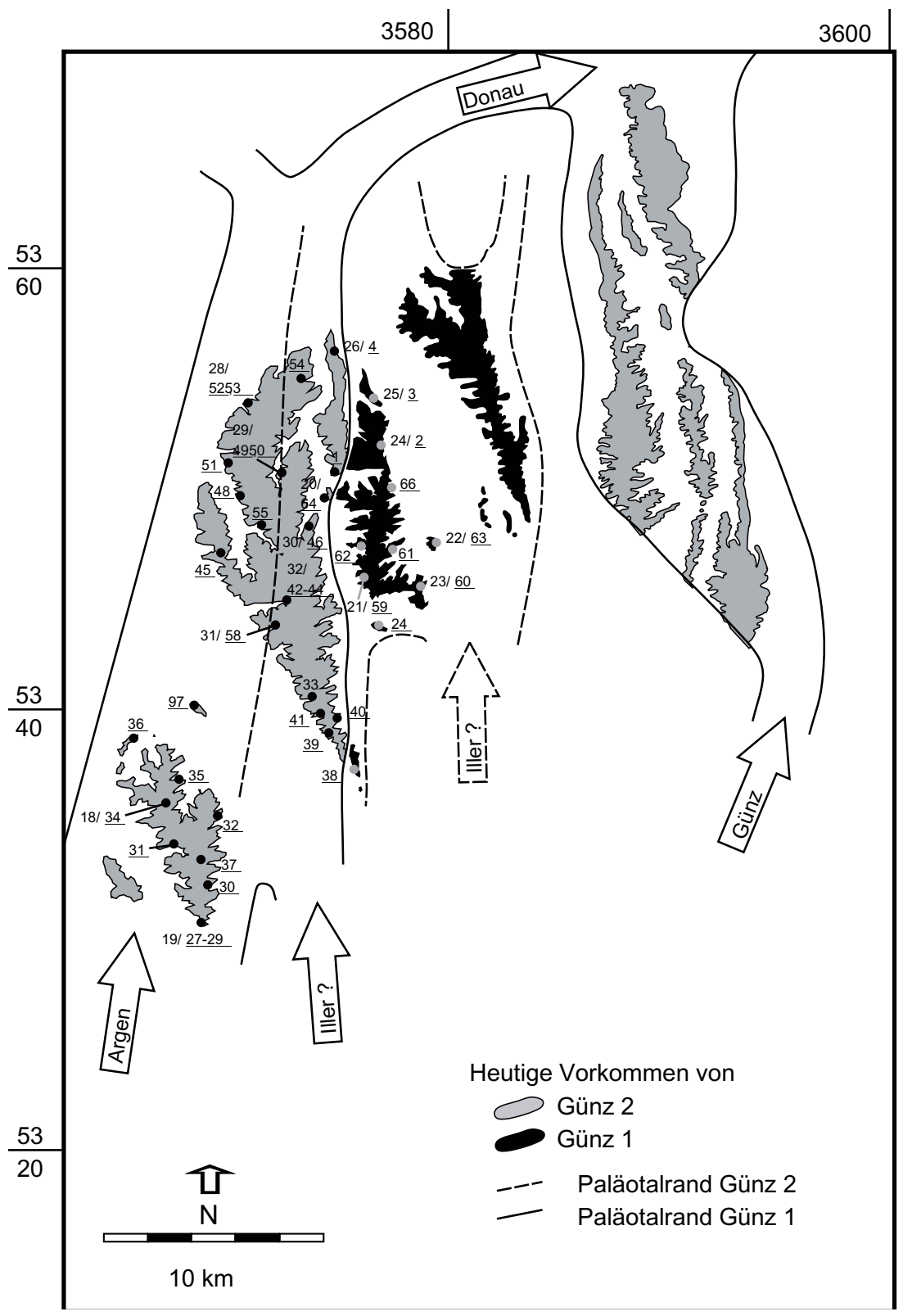

Abb. 8: Talverlauf zur Zeit der Ablagerung von Günz 1 und 2. Die Ziffern geben die Probenentnahmestellen für petrographische Analysen (ohne Unterstrich, Nummerierung nach Tab. 2) und Schwermineraluntersuchungen (mit Unterstrich, Nummerierung nach Tab. 3) an.

Fig. 8: Valley from Günz 1 to Günz 2. The sample locations are indicated by underlined numbers for petrographic analyses (tab. 2) and numbers for heavy mineral analyses (tab. 3). 
Westlich und östlich dieser Terrasse sind die Günz 2-zeitlichen Vorkommen auf dem Heggbacher Feld, dem Holzstöcke-Feld und dem Weißenhorn-Kissendorfer Feld erhalten. Die Täler von Argen und Iller verliefen voneinander getrennt und mündeten bei Ulm bzw. Leipheim in die Donau. Für die stratigraphische Zuordnung ist die räumliche Beziehung zum Zeiler Schotter wesentlich, das zuletzt von SCHREINER (2003) beschrieben und als Typregion für die Günzeiszeit vorgeschlagen worden ist. Der Zeiler Schotter lässt sich mit 4,3\%o mit den Vorkommen bei Schwendi verknüpfen, jedoch bleibt wegen der großen Entfernung, über die interpoliert werden muss, eine gewisse Unsicherheit bestehen. Die Korrelation steht jedoch im Einklang mit den Überlegungen von DOPPLER (2003) und wird auch durch die geröllpetrographischen Ergebnisse (durchschnittlicher Kristallingehalt: 2,5\%) gestützt.

Die Ergebnisse zeigen, dass die petrographischen Daten die geschilderte flussgeschichtliche Interpretation stützen und im Zusammenhang mit den modellierten Paläotälern zu einem schlüssigen Bild der unterpleistozänen Landschaftsgeschichte führen. Jedes der in den Abbildungen 6 bis 8 dargestellten Täler ist durch eine petrographische Ausbildung ausgewiesen, die im Rahmen der statistischen Fehler und bei Berücksichtigung von Verwitterungseffekten homogen ist. Eine gegenseitige Unterscheidbarkeit der glazifluviatilen Terrassenschotter aufgrund der Petrographie gelingt jedoch nicht. Der älteste Periglazialschotter der Argenschüttung ist gegenüber den nachfolgenden glazifluviatilen Terrassenschottern im Riß-Iller-Gebiet durch eine eindeutige Signatur im Geröll- und Schwermineralspektrum ausgezeichnet. Erst östlich einer Linie Memmingen - Leipheim ist eine Differenzierung der donauzeitlichen Einheiten aufgrund des Dolomit- und Kristallingehaltes nachweisbar (LÖSCHER 1976).

\section{Dank}

Bei den Herrn Dr. G. Doppler sowie Dr. C. Hoselmann bedanken wir uns herzlich für die Anregungen, die zur Verbesserung des Manuskripts beigetragen haben.

\section{Literatur}

Becker-Haumann，R. (1995): Zur Flußgeschichte der ältestpleistozänen Iller. - Sonderveröffentlichungen des Geologischen Institutes der Universität zu Köln, 98: 168 S.; Köln.

Becker-Haumann, R. (1996): Die Petrologie der kristallinen Iller-Gerölle (bayerisches Alpenvorland). - Aufschluss, 46: 13-28; Heidelberg.

Becker-Haumann, R. (2007): Hydraulic parameters as key factors for the 3D-modeling of fluvial deposits. - Transactions in GIS, 11(1): 83-100; Blackwell.

Becker-Haumann，R. (2005): Anwendungen der Geoinformatik für die hochauflösende 3D-Modellierung fluviatiler Terrassenkörper. - 332 S.; Stuttgart (Schweizerbart).

Becker-Haumann, R. \& Gerth, A. (im Druck): 3D-Modellierung pleistozäner Schmelzwasserabflüsse im Verzahnungsbereich von Rheinund Illergletscher, deutsches Alpenvorland. - Zeitschrift für Geomorphologie (zum Druck angenommen).

Boenigk, W. (1983): Schwermineralanalyse. - 158 S.; Stuttgart (Enke).

Deutsche Stratigraphische Kommission (2002): Stratigraphische Tabelle von Deutschland 2002. -16 S.; Potsdam.

Doppler, G. (2003): Zur Gliederung von Ältesten Periglazialschottern und Älteren Deckenschottern im Rot-Günz-Gebiet (Oberschwaben und Bayerisch Schwaben). - Zeitschrift der Deutschen Geologischen Gesellschaft, 154/2-3: 255286; Stuttgart.

EBerhard, M. (1986): Litho- und Biostratigraphie im Oberen Süßwassermolasse-Fächer der Adelegg (Südbayern). - Jahrbuch Geol. B. Reihe A, 129: 5-39; Wien.

EBERHARD, M. (1987): Entwicklung von Sedimentation, Flora, Fauna, Klima und Relief von Mittelmiozän bis Quartär zwischen Arlberg (Vorarlberg / Tirol) und Adelegg (Allgäu). - Mitteilung des Geologischen Instituts der ETH u. Univ. Zürich, N. F., 267: 242 S.; Zürich.

EBerl, B. (1930): Die Eiszeitenfolge im nördlichen Alpenvorlande. - 427 S.; Augsburg (Filser).

EichleR, H. (1970): Das präwürmzeitliche Pleistozän zwischen Riss und oberer Rottum. - Heidelberger Geographische Arbeiten, 30: 128 S.; Heidelberg. 
Ellwanger, D., Bibus, E., Bludau, W., Kösel, M. \& Merkt, J. (1995): XI. Baden-Württemberg. - In: Benda, L. (Hrsg.): Das Quartär Deutschlands: 255-295; Stuttgart (Bornträger).

Fezer, F. (1969): Tiefenverwitterung circumalpiner Pleistozänschotter. - Heidelberger Geographische Arbeiten, 24: 144 S.; Heidelberg.

Geiger, E. (1969): Der Geröllbestand des Rheingletschergebietes nördlich von Bodensee und Rhein. - Jahreshefte des Geologischen Landesamts Baden-Württ., 11: 127-172; Freiburg i. Br.

Graul, H. (1953): Über die quartären Geröllfazien im deutschen Alpenvorlande. - Geologica Bavarica, 19: 266-280; München.

Graul, H. (1962) Eine Revision der pleistozänen Stratigraphie des schwäbischen Alpenvorlandes. - Petermanns Geographische Mitteilungen, 106: 253-271; Gotha.

GRAUL, H. (1968): Führer zur zweitägigen Exkursion im nördlichen Rheingletschergebiet - August 1968. - Heidelberger Geographische Arbeiten, 49: 31-75; Heidelberg.

HAAG, T. (1982): Das Mindelglazial des nordöstlichen Rheingletschers zwischen Riß und Iller. - Jber. Mitt. oberrhein. geol. Ver., N. F., 64: 225-266; Stuttgart.

HabBe, K. A. (2003): Gliederung und Dauer des Pleistozäns im Alpenvorland, in Nordwesteuropa und im marinen Bereich. - Bemerkungen $\mathrm{zu}$ einigen neueren Korrelierungsversuchen. - Zeitschrift der Deutschen Geologischen Gesellschaft, 154/2-3: 171-192; Stuttgart.

Halder, W. (1988): Die Obere Süßwassermolasse des Hochgratfächers. - Arbeiten des Inst. für Geologie und Paläontologie der Universität Stuttgart, N. F., 84: 121-171; Stuttgart.

Hansel, H (1989): Erläuterungen zu Blatt 8226 Isny Nord. - Geol. Kt. Baden-Württ. 1:25000: 89 S.; Stuttgart.

Jerz, H. (1995): XII. Bayern. - In: Benda, L. (Hrsg.): Das Quartär Deutschlands: 296-326; Stuttgart (Borntraeger).

Litt, T., Ellwanger, D., Villinger, E. \& Wansa, S. (2005): Das Quartär in der Stratigraphischen Tabelle von Deutschland 2002. - Newsl. Stratigr., 41: 385-399; Berlin.

LösCHER, M. (1976): Die präwürmzeitlichen Schotterablagerungen in der nördlichen Iller-LechPlatte. - Heidelberger Geographische Arbeiten, 45: IX+157; Heidelberg.
Löscher, M., Münzing, K., Tillmanns, W. (1978): Zur Paläogeographie der nördlichen Iller-LechPlatte und zur Genese ihrer Schotter im Altpleistozän. - Eiszeitalter und Gegenwart, 28: 68-82; Öhringen.

Penck, A. \& Brǘckner, E. (1901-1909): Die Alpen im Eiszeitalter. - 2 Bde., 1199 S.; Leipzig.

RöGNER, K. (1986): Genese und Stratigraphie der ältesten Schotter der südlichen Iller-Lechplatte (Bayerisch-Schwaben). - Eiszeitalter und Gegenwart, 36: 111-119; Hannover.

SCHÄDEL, K. (1952): Die Stratigraphie des Altdiluviums im Rheingletschergebiet. - Jber. Mitt. oberrhein. geol. Ver., N.F., 34: 1-20; Stuttgart.

Schädel, K. \& Werner, J. (1963): Neue Gesichtspunkte zur Stratigraphie des mittleren und älteren Pleistozäns im Rheingletschergebiet. - Eiszeitalter und Gegewart 14: 5-26; Öhringen.

Schaefer, I. (1995): Das Alpenvorland im Zenit des Eiszeitalters. - Forschungen in seinem Kerngebiet zwischen Riß und Lech. - Textband. 405 S.; Stuttgart (Steiner).

SCHLÜChtER, C. (1989): A non-classical summary of the Quaternary stratigraphy in the Northern Alpine Foreland of Switzerland. - Bull. de la Soc. neuchâteloise de géogr., 32-33: 143-157; Neuchâtel.

SchreIner, A. (1992): Einführung in die Quartärgeologie. - 258 S.; Stuttgart (Schweizerbart).

SchreIner, A. \& Ebel, R. (1981) Quartärgeologische Untersuchungen in der Umgebung von Interglazialvorkommen im östlichen und südlichen Illergletscher-Vorland. - Heidelberger Geographische Arbeiten, 37: 159 S.; Heidelberg.

SinN, P. (1972): Zur Stratigraphie und Paläogeographie des Präwürm im mittleren und südlichen Illergletschervorland. - Heidelberger Geographische Arbeiten, 37: IX +159 S.; Heidelberg.

TAnner, H (1944): Beitrag zur Geologie der Molasse zwischen Ricken und Hörnli. - Mitt. thurg. naturforsch. Ges., 33: 5-108; Frauenfeld.

VILlinger, E. (2003): Zur Paläogeographie von Alpenrhein und oberer Donau. - Zeitschrift der Deutschen Geologischen Gesellschaft, 154/2-3: 193-253; Stuttgart.

Weidenbach, F. (1937): Bildungsweise und Stratigraphie der diluvialen Ablagerungen Oberschwabens. - N. Jb. Mineral. u. Geol., Beil., 78, Abt. B: 66-108; Stuttgart.

Weidenbach, F. (1940): Blatt Ochsenhausen Nr. 
158. - Erl. geol. Spezialkarte Württ. - 63 S.; Stuttgart.

WeYL, R. (1952): Schwermineraluntersuchungen an eiszeitlichen Ablagerungen der Riß-Lech-Platte.

- Geologica Bavarica, 14: 107-123; München. 


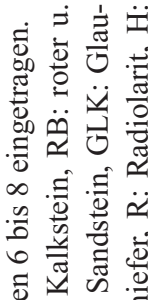

क्ष $-\overline{0}$

总㐫

䨌

ष

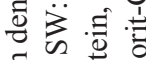

$\exists$ 舫

믈 잉

क ज्ञ

ల్

焉它的

일

ป స्ञ

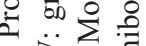

。

○ิ

कि

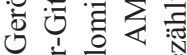

¿ ब.

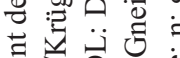

总瑝

巳 无艺胥

ग)

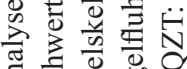

过

플 즐

苍获芯志

है

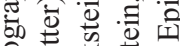

过节泀

离

过苛离

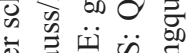

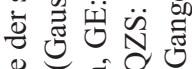

क

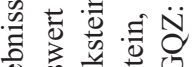

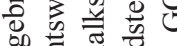

可 늘

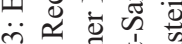

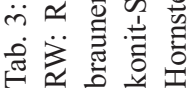

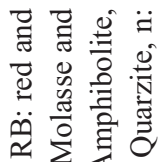

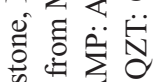

\& $2 \ll$

ఏ司

造过武

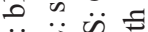

芦范竞

थ

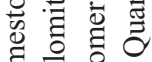

河 弟

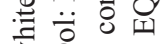

b

.

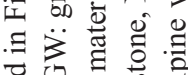

రृ

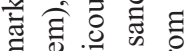

ฮ

㐘交产

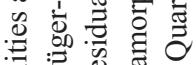

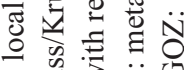

至

हृ

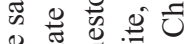

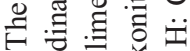

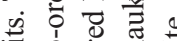

要

穴

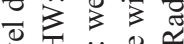

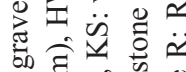

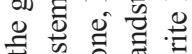

额总

㐫造艺

.일

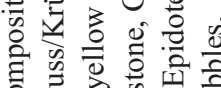
ᄋ

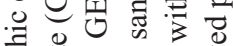

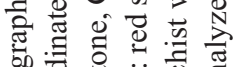

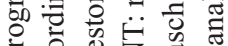
苟 ค.

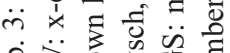

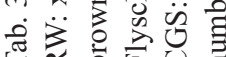

\begin{tabular}{|c|c|c|c|c|c|c|c|c|c|c|c|c|c|c|c|c|}
\hline & $\vec{m}$ & $0^{\circ}$ & $\stackrel{\sigma}{m}$ & n) & $\begin{array}{l}0 \\
0\end{array}$ & $\stackrel{\forall}{\forall}$ & $\begin{array}{c}\text { क) } \\
10\end{array}$ & $\stackrel{m}{=}$ & $\hat{0}$ & $\begin{array}{l}m \\
\stackrel{0}{\circ}\end{array}$ & $\stackrel{\infty}{\infty}$ & $\stackrel{\infty}{+}$ & $\begin{array}{l}+ \\
\infty\end{array}$ & $\bar{\sigma}$ & ウ் & $\sigma$ \\
\hline & क & $\stackrel{0}{0}$ & $\begin{array}{l}0 \\
0\end{array}$ & $\stackrel{\oplus}{\tilde{m}}$ & $\overbrace{0}^{+}$ & $\infty$ & $\nabla$ & $\frac{\mathscr{\sigma}}{\sigma^{\circ}}$ & 5 & $\hat{m}$ & $\bar{\sigma}$ & $\bar{\sim}$ & $\begin{array}{l}\infty \\
\infty \\
\sigma^{-}\end{array}$ & $\stackrel{m}{m}$ & m & $\stackrel{m}{\sim}$ \\
\hline & $\overline{\bar{\omega}}$ & $\stackrel{\circ}{\circ}$ & $\stackrel{\nabla}{N}$ & $\stackrel{ }{\circ}$ & $\stackrel{N}{N}$ & $\hat{6}$ & $\begin{array}{l}\stackrel{\infty}{\circ} \\
\infty\end{array}$ & $\stackrel{\infty}{\infty}$ & 6 & $\stackrel{\sim}{\stackrel{0}{\sim}}$ & $\begin{array}{l}10 \\
\infty \\
\infty\end{array}$ & $\stackrel{\vec{m}}{\sim}$ & $\begin{array}{l}0 \\
\stackrel{0}{\circ} \\
-\end{array}$ & $\begin{array}{l}10 \\
\infty \\
\infty\end{array}$ & m. & $\stackrel{\Omega}{N}$ \\
\hline & ๘ & 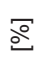 & $\bar{N}$ & న & $\infty$ & $\begin{array}{l}\text { @ } \\
\stackrel{\infty}{\infty}\end{array}$ & $\underset{\infty}{N}$ & $\Re$ & $\stackrel{0}{N}$ & $\tilde{N}^{\infty}$ & \begin{tabular}{l}
0 \\
\multirow{0}{*}{}
\end{tabular} & $\infty$ & $\begin{array}{l}m \\
\stackrel{n}{R}\end{array}$ & $\bar{\sigma}$ & $\stackrel{L}{R}$ & $\frac{\infty}{\infty}$ \\
\hline & ᄃ & & $\infty$ & $\frac{\nabla}{m}$ & $\stackrel{\sim}{\sim}$ & ம & 离 & $\underset{\nearrow}{\check{y}}$ & $\begin{array}{l}\text { న } \\
\text { ల్ల }\end{array}$ & స్ల & §̃ & 只 & $\bar{m}$ & 六 & $\frac{10}{m}$ & ᄋ్ల \\
\hline & ๖ั & ¿ & $\stackrel{m}{\sim}$ & $\stackrel{N}{\sim}$ & $m$ & $\stackrel{\sigma}{\sim}$ & $\stackrel{\nabla}{\sim}$ & $\stackrel{\sim}{N}$ & $\stackrel{\vec{\sigma}}{\dot{m}}$ & $\stackrel{\text { ఠ }}{\forall}$ & ñ & $\stackrel{n}{\sim}$ & $\begin{array}{l}10 \\
10\end{array}$ & $\stackrel{N}{\tilde{n}}$ & $\stackrel{10}{n}$ & $\stackrel{0}{N}$ \\
\hline $\begin{array}{l}\frac{5}{\text { II }} \\
\text { I. }\end{array}$ & 정 & $\overbrace{}^{\circ}$ & $\stackrel{N}{\sim}$ & $\begin{array}{l}\infty \\
0^{-}\end{array}$ & $\stackrel{10}{\sim}$ & $\begin{array}{l}\infty \\
0^{-}\end{array}$ & $\bar{i}$ & $\stackrel{\nabla}{\dot{m}}$ & $\stackrel{\nabla}{r}$ & $\stackrel{0}{\sim}$ & $\stackrel{N}{=}$ & 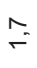 & $\begin{array}{l}0 \\
0 \\
0\end{array}$ & $\begin{array}{l}0 \\
0 \\
0\end{array}$ & $\stackrel{\Delta}{\sim}$ & $\underset{0}{N}$ \\
\hline & $x$ I & $\stackrel{\circ}{\circ}$ & $\sigma_{0}^{\circ}$ & $\stackrel{10}{\sim}$ & $\bar{i}$ & 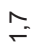 & $\stackrel{+}{\sim}$ & $\hat{N}$ & $\stackrel{\leftrightarrow}{\sim}$ & $\stackrel{\infty}{\sim}$ & $\bar{m}$ & o & $\stackrel{m}{\sim}$ & $\underset{\sigma 0}{t}$ & $\begin{array}{l}10 \\
m\end{array}$ & $\stackrel{\sim}{\sim}$ \\
\hline & 号 & $\stackrel{8}{2}$ & 0 & 0 & $\begin{array}{l}\infty \\
\infty \\
0^{-}\end{array}$ & $\stackrel{\infty}{\infty}$ & $\stackrel{N}{\sim}$ & 0 & $\stackrel{0}{0}$ & 0 & 0 & 0 & $\stackrel{\infty}{\infty}$ & 0 & $\stackrel{N}{\sim}$ & 0 \\
\hline$\frac{\underline{\underline{\underline{E}}}}{\overline{\bar{g}}}$ & D & $\overbrace{}^{\circ}$ & $\mid \begin{array}{l}\infty \\
0 \\
0\end{array}$ & 0 & $\stackrel{N}{\sim}$ & $\stackrel{\nabla}{\sim}$ & $\begin{array}{l}\infty \\
0^{-}\end{array}$ & $\stackrel{\infty}{\leftarrow}$ & の & $\hat{0}$ & 0 & 0 & 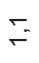 & $\begin{array}{l}10 \\
0 \\
0\end{array}$ & $\stackrel{m}{\sim}$ & 0 \\
\hline$\geq$ & $\sum_{<}^{n}$ & 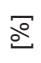 & $\stackrel{0}{\sim}$ & $\stackrel{N}{=}$ & $\stackrel{0}{r}$ & $\stackrel{m}{=}$ & $\begin{array}{l}\infty \\
0^{-}\end{array}$ & $\stackrel{\nabla}{\sim}$ & $\hat{0}$ & $\stackrel{\infty}{-}$ & $\stackrel{\vec{m}}{m^{\prime}}$ & $\stackrel{+}{\circ}$ & $\stackrel{10}{=}$ & $\hat{0}$ & $\stackrel{10}{=}$ & $\underset{0}{N}$ \\
\hline & $\sum_{0}^{\infty}$ & 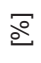 & $\stackrel{N}{m}$ & $\stackrel{d}{i}$ & $\stackrel{\infty}{\sim}$ & م) & $\stackrel{\sim}{\sim}$ & $\hat{\sim}$ & $\stackrel{\leftrightarrow}{\sim}$ & $\stackrel{\sim}{\sim}$ & $\stackrel{2}{\omega^{\circ}}$ & 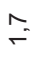 & $\stackrel{+}{\sim}$ & $\bar{i}$ & $\stackrel{m}{\sim}$ & $\check{\sigma}$ \\
\hline & 崩 & $\stackrel{2}{\circ}$ & 0 & 0 & 0 & 0 & 0 & 0 & 0 & 0 & 0 & 0 & $\tilde{O}$ & 0 & 0 & 0 \\
\hline & N & 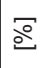 & $\stackrel{\Delta}{\sim}$ & $\begin{array}{l}\nabla_{\infty} \\
\infty\end{array}$ & $\stackrel{+}{0}$ & $\stackrel{m}{n}$ & $\hat{\sigma}^{\circ}$ & $\begin{array}{l}\infty \\
\sigma^{-}\end{array}$ & 음 & 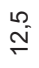 & $\begin{array}{l}10 \\
\infty \\
\infty\end{array}$ & $\bar{m}$ & $\stackrel{\Omega}{N}$ & $\underset{\sigma^{\circ}}{N}$ & $\infty$ & $\stackrel{\Omega}{N}$ \\
\hline 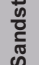 & 竎 & $\stackrel{2}{2}$ & 0 & $\stackrel{\sim}{\sim}$ & $\begin{array}{l}0 \\
0\end{array}$ & 7 & $\stackrel{\infty}{-}$ & 0 & $0^{\circ}$ & 0 & 0 & 0 & $\stackrel{+}{\sim}$ & $\bar{m}$ & $\stackrel{m}{r}$ & 0 \\
\hline & Ł & ¿ & 0 & $\begin{array}{l}\sigma_{0} \\
\sigma^{\prime}\end{array}$ & 0 & 0 & $\stackrel{+}{\circ}$ & 0 & 0 & 0 & 0 & 0 & 0 & $\underset{\sigma}{N}$ & 0 & 0 \\
\hline & $\stackrel{\vec{O}}{\Sigma}$ & ¿ & 0 & 0 & $\underset{\sigma}{N}$ & 10 & 0 & 0 & 0 & 0 & 0 & 0 & 0 & 0 & 0 & 0 \\
\hline $\begin{array}{l}\dot{\bar{\circ}} \\
\stackrel{=}{=}\end{array}$ & Oे & হ & 0 & 0 & 0 & 10 & 0 & 0 & 0 & 0 & 0 & 0 & 0 & 0 & 0 & 0 \\
\hline & $\stackrel{\mathscr{N}}{\mathscr{x}}$ & 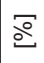 & $\stackrel{m}{=}$ & $\stackrel{\stackrel{N}{m}}{\stackrel{m}{\sim}}$ & $\nabla_{-}$ & I & $\begin{array}{l}\stackrel{2}{0} \\
\stackrel{5}{\leftarrow}\end{array}$ & $\stackrel{m}{\tilde{N}}$ & & $\stackrel{\sim}{\stackrel{\sim}{\sim}}$ & $\bar{i}$ & $\bar{v}$ & $\stackrel{10}{\infty}$ & $\begin{array}{l}m \\
\stackrel{\infty}{\circ}\end{array}$ & 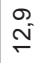 & $\stackrel{\sim}{ }$ \\
\hline & 凹 & $\overbrace{}^{\circ}$ & ले & $\stackrel{0}{N}$ & $10^{\circ}$ & $\nabla$ & $\begin{array}{l}m \\
\infty\end{array}$ & $\stackrel{0}{\tilde{m}^{-}}$ & 6 & $\begin{array}{l}\text { क) } \\
\infty\end{array}$ & $\stackrel{m}{\sim}$ & $\stackrel{n}{n}$ & $\begin{array}{l}0 \\
\sigma^{\circ}\end{array}$ & ஸू & $\bar{m}$ & $\underset{60}{\vec{v}}$ \\
\hline $\begin{array}{l}\tilde{n} \\
\stackrel{\text { D }}{=}\end{array}$ & $\ddot{q}$ & $\stackrel{2}{\circ}$ & ร & $\underset{\omega}{N}$ & $\stackrel{0}{N}$ & $\infty$ & No & $\stackrel{\sim}{\sim}$ & m. & $\stackrel{0}{\sim}$ & $\stackrel{\nabla}{\sigma}$ & $\stackrel{m}{\sim}$ & $\bar{m}$ & $\hat{m}$ & in & $\underset{10}{*}$ \\
\hline & ळ & $\stackrel{2}{2}$ & $m$ & $\stackrel{\sim}{\sim}$ & $\stackrel{\dot{v}}{\mathrm{i}}$ & N & $\begin{array}{l}10 \\
\tilde{n}\end{array}$ & 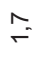 & N & $\stackrel{N}{\sim}$ & $\underset{\sim}{N}$ & $\stackrel{\sim}{\sim}$ & $\stackrel{\Omega}{\circ}$ & $\stackrel{\sigma}{-}$ & $\stackrel{\rho}{\sim}$ & $\stackrel{N}{m}$ \\
\hline & 夜 & $\overbrace{}^{\circ}$ & 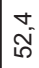 & $\hat{\kappa}$ & $N$ & 0 & $\hat{\sigma}$ & $\stackrel{\sim}{\widetilde{\gamma}}$ & & 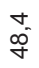 & $\begin{array}{l}0 \\
\text { in }\end{array}$ & $\stackrel{\check{\sim}}{\stackrel{\sim}{*}}$ & $\frac{m}{6}$ & $\begin{array}{l}m \\
\hat{\sigma}^{\circ}\end{array}$ & $\begin{array}{l}0 \\
\dot{0} \\
\text { in }\end{array}$ & $\stackrel{\infty}{\mathcal{\sigma}}$ \\
\hline & $\stackrel{m}{a}$ & $\stackrel{!}{a}$ & $r$ & $N$ & $m$ & $\nabla$ & ما & 0 & $\lambda$ & $\infty$ & $\sigma$ & $\leftarrow$ & $\mp$ & $\stackrel{N}{\sim}$ & $\stackrel{m}{\sim}$ & $\stackrel{\Xi}{\leftarrow}$ \\
\hline & & & & & & & & & & & & & & & & \\
\hline
\end{tabular}




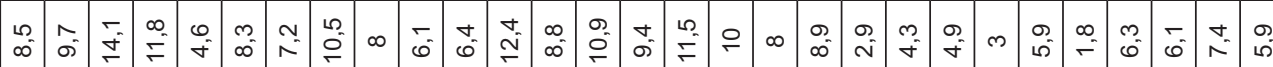
A

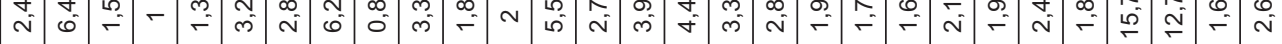

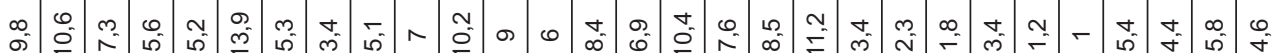

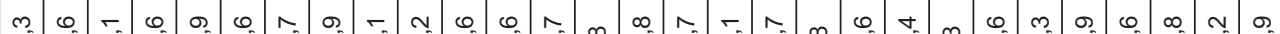

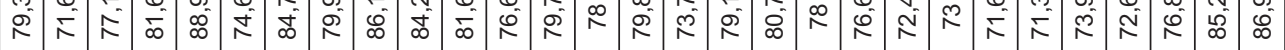

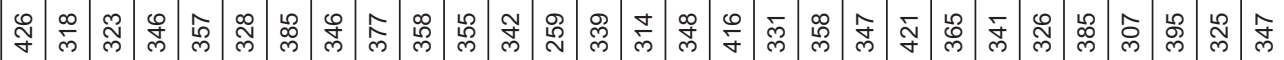

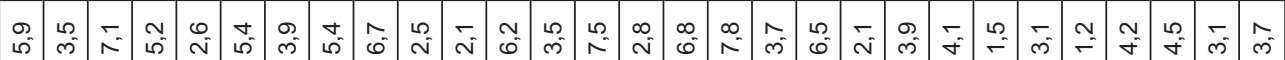

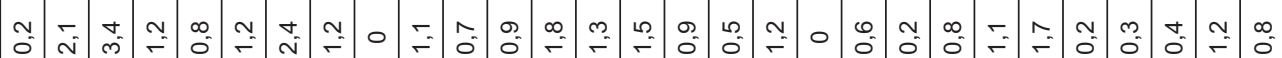

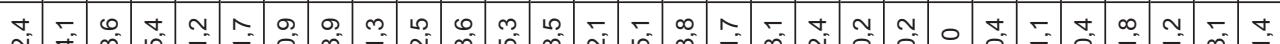

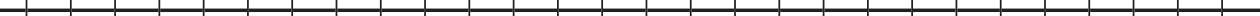

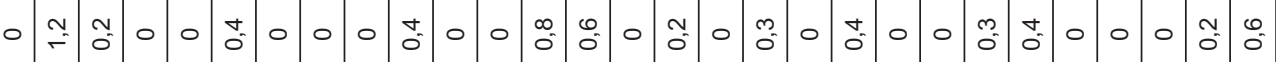

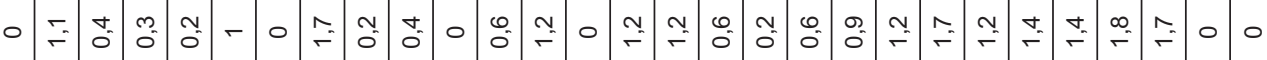

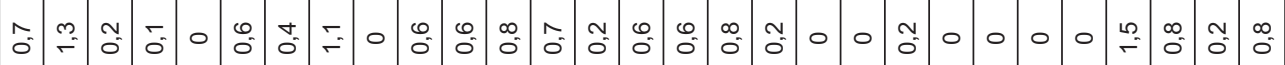

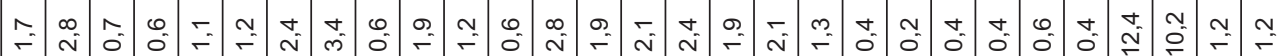

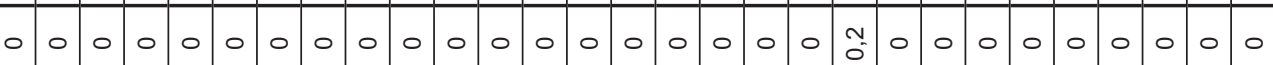

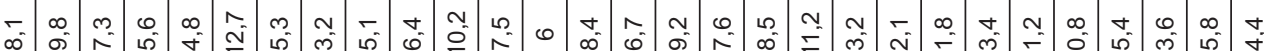

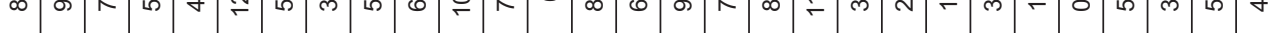

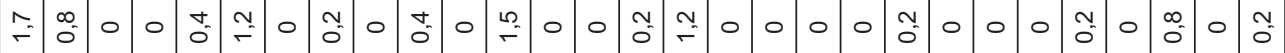

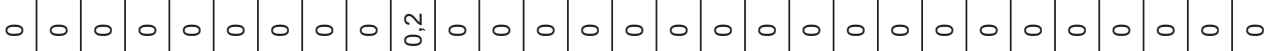
$\begin{array}{lllllllllllllllllllllllllllll}0 & 0 & 0 & 0 & 0 & 0 & 0 & 0 & 0 & 0 & 0 & 0 & 0 & 0 & 0 & 0 & 0 & 0 & 0 & 0 & 0 & 0 & 0 & 0 & 0 & 0 & 0 & 0 & 0\end{array}$

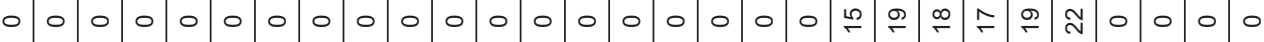

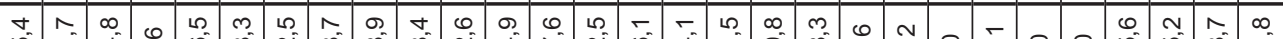

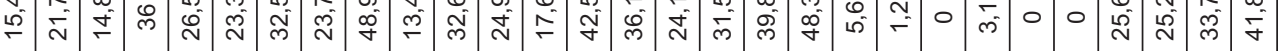

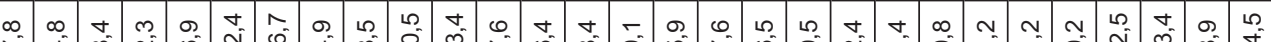

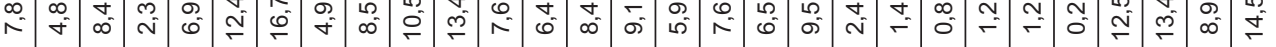
N 0 c

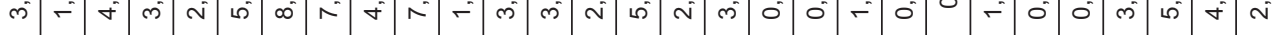

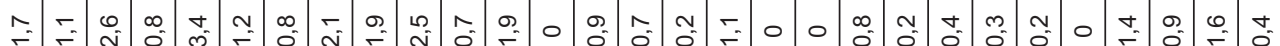

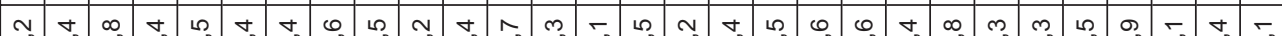

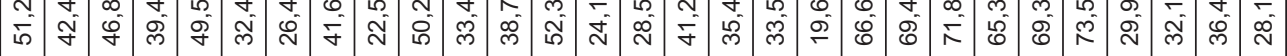

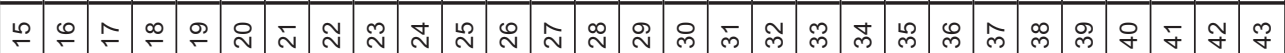




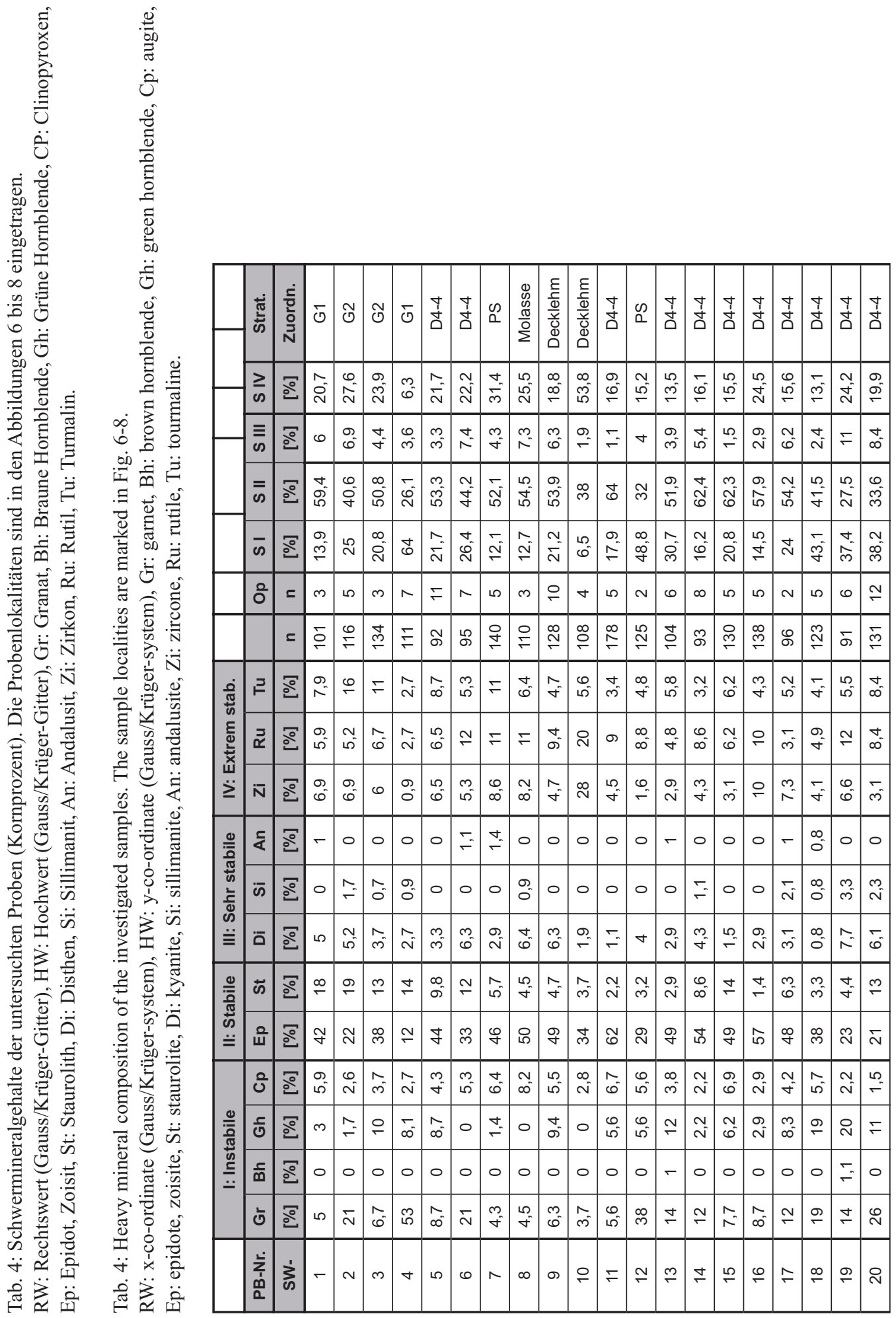




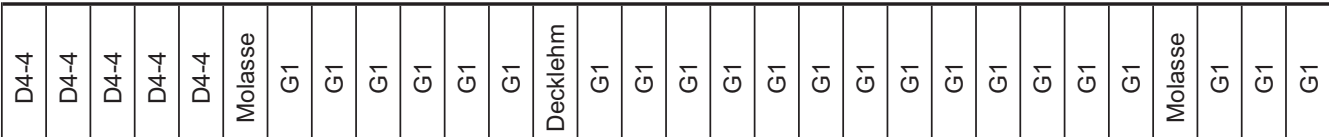

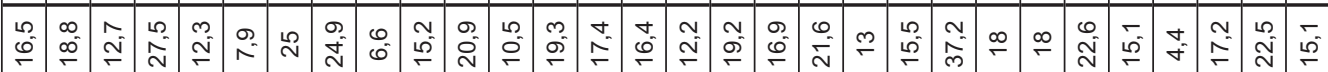

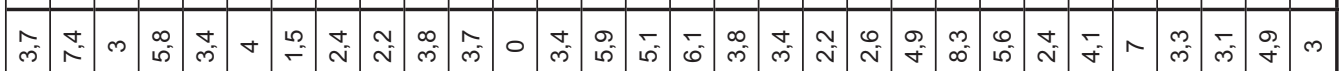

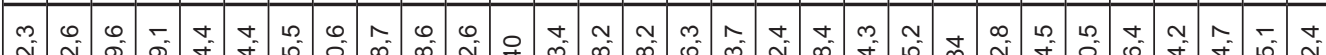

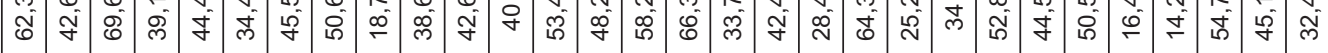

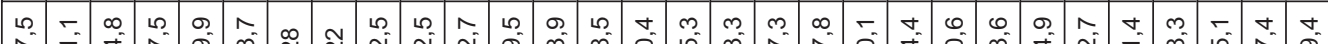

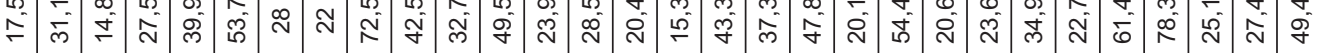

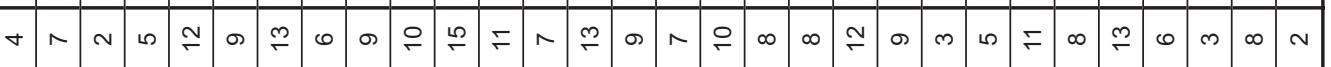

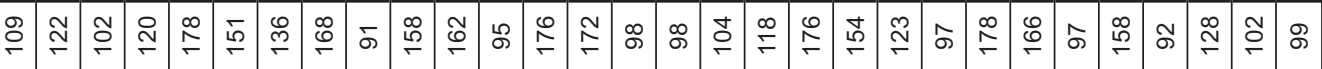

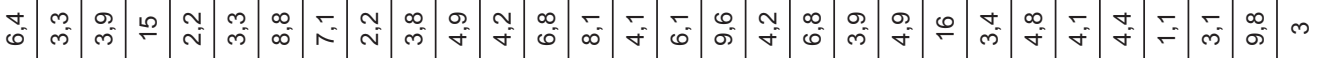

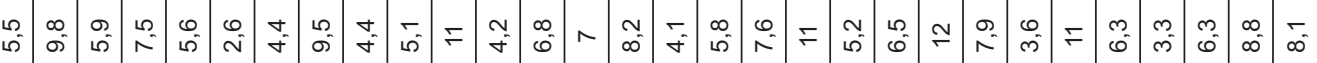

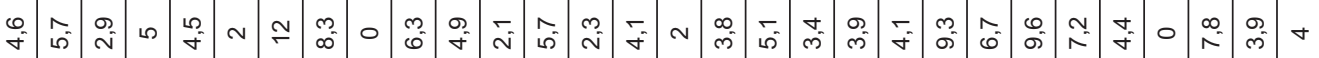

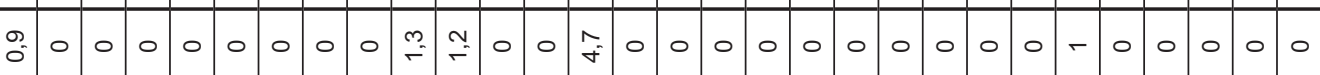

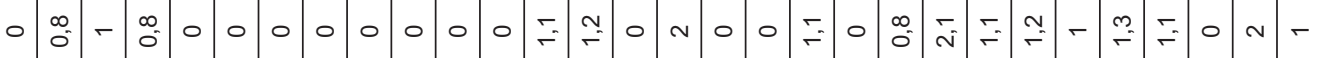

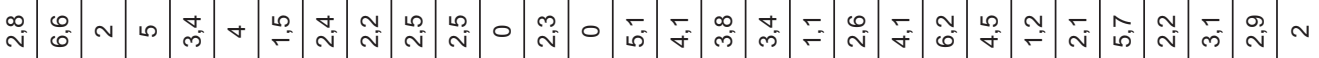

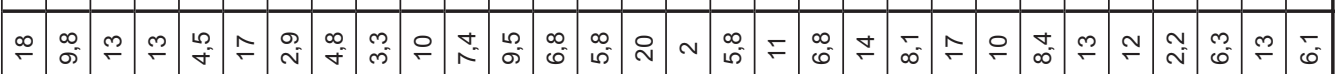
J

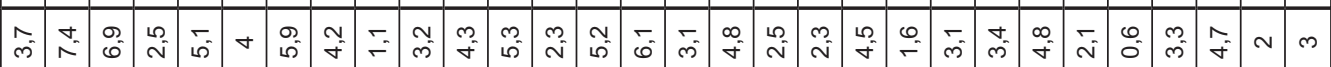

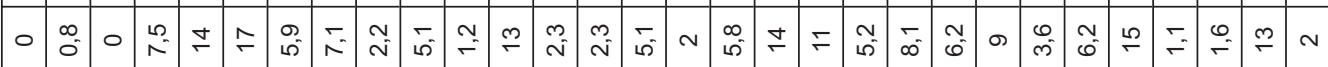

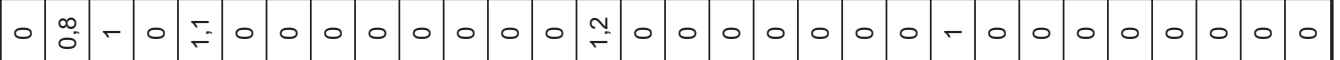

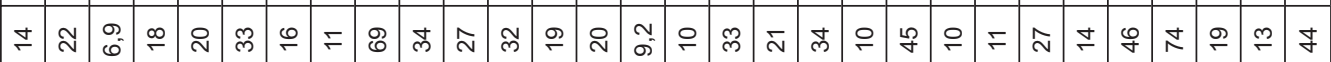

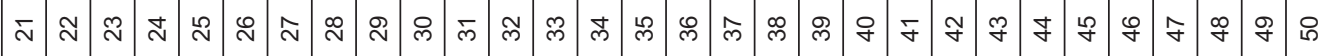




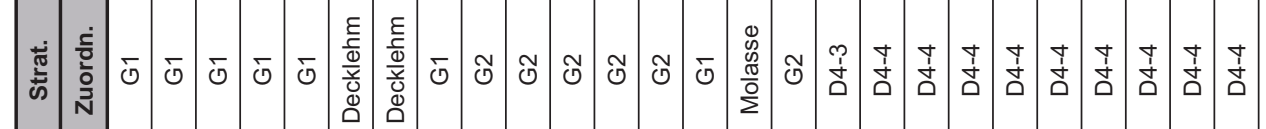

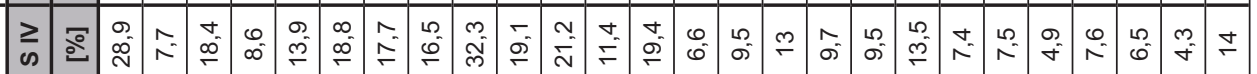

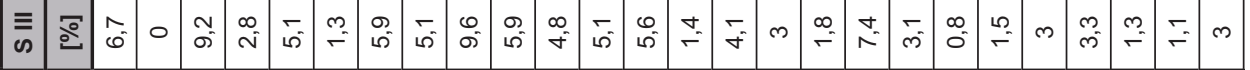

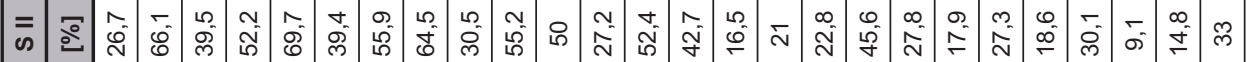

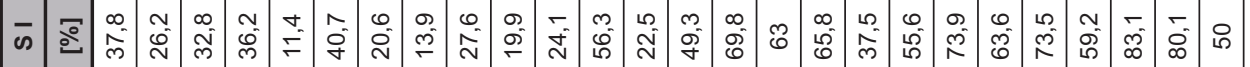

ठำ = = ৪

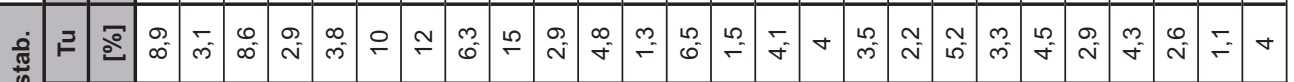

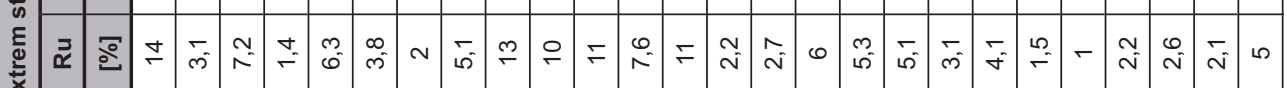

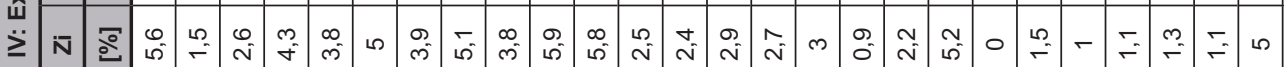

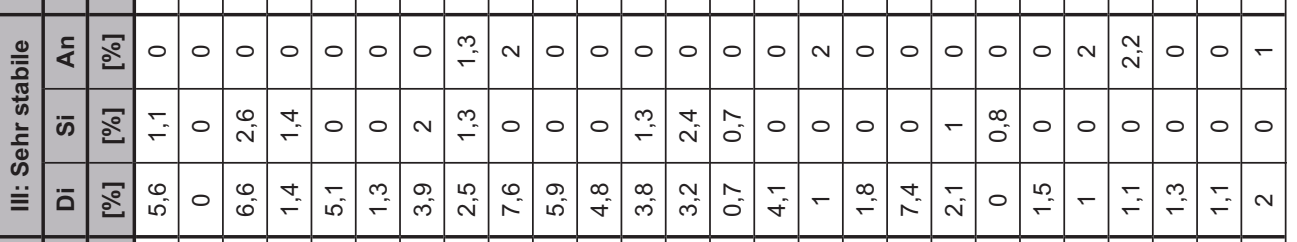




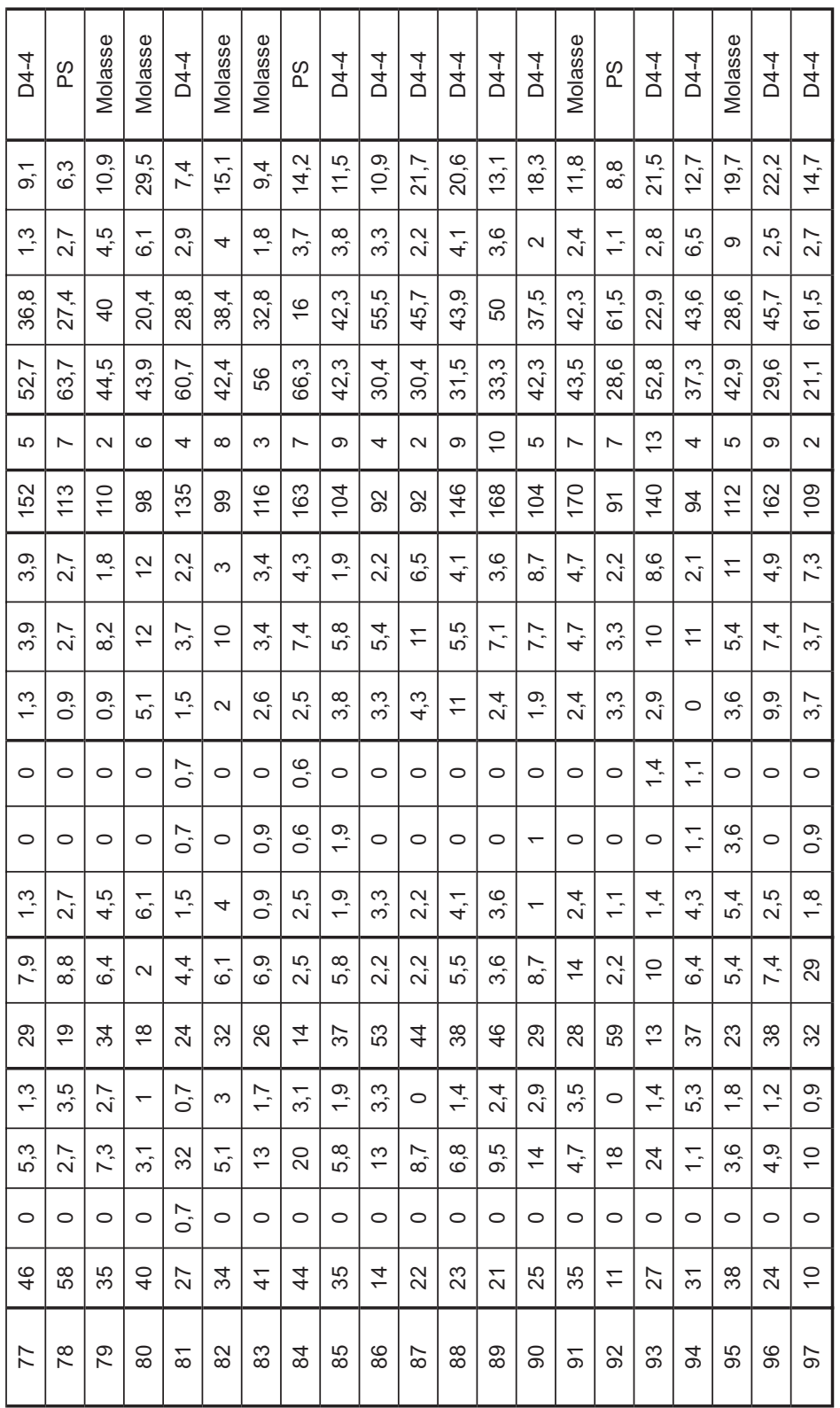

